\title{
In silico Analysis of HIV-1 Env-gp120 Reveals Structural Bases for Viral Adaptation in Growth-Restrictive Cells
}

\author{
Masaru Yokoyama ${ }^{1 \dagger}$, Masako Nomaguchi2t, Naoya Doi ${ }^{2}$, Tadahito Kanda ${ }^{1,3}$, \\ Akio Adachi ${ }^{2 *}$ and Hironori Sato ${ }^{1 *}$ \\ ${ }^{1}$ Laboratory of Viral Genomics, Pathogen Genomics Center, National Institute of Infectious Diseases, Tokyo, Japan, \\ ${ }^{2}$ Department of Microbiology, Institute of Biomedical Sciences, Tokushima University Graduate School, Tokushima, Japan, \\ ${ }^{3}$ Department of Research Promotion, Division of Infectious Disease Research, Japan Agency for Medical Research and \\ Development, Tokyo, Japan
}

OPEN ACCESS

Edited by:

Katsumi Maenaka,

Hokkaido University, Japan

Reviewed by:

Yong-Hui Zheng,

Michigan State University, USA

Naoyuki Miyashita,

Kindai University, Japan

${ }^{*}$ Correspondence:

Akio Adach

adachi@tokushima-u.ac.jp;

Hironori Sato

hirosato@nih.go.jp

tThese authors have contributed equally to this work.

Specialty section: This article was submitted to

Virology,

a section of the journa

Frontiers in Microbiology

Received: 14 October 2015

Accepted: 21 January 2016

Published: 09 February 2016

Citation:

Yokoyama M, Nomaguchi M, Doi N, Kanda T, Adachi A and Sato H (2016) In silico Analysis of HIV-1 Env-gp120 Reveals Structural Bases for Viral Adaptation in Growth-Restrictive

Cells. Front. Microbiol. 7:110. doi: 10.3389/fmicb.2016.00110
Variable V1/N2 and V3 loops on human immunodeficiency virus type 1 (HIV-1) envelopegp120 core play key roles in modulating viral competence to recognize two infection receptors, CD4 and chemokine-receptors. However, molecular bases for the modulation largely remain unclear. To address these issues, we constructed structural models for a full-length gp120 in CD4-free and -bound states. The models showed topologies of gp120 surface loop that agree with those in reported structural data. Molecular dynamics simulation showed that in the unliganded state, V1/V2 loop settled into a thermodynamically stable arrangement near $\mathrm{V} 3$ loop for conformational masking of V3 tip, a potent neutralization epitope. In the CD4-bound state, however, V1/V2 loop was rearranged near the bound CD4 to support CD4 binding. In parallel, cell-based adaptation in the absence of anti-viral antibody pressures led to the identification of amino acid substitutions that individually enhance viral entry and growth efficiencies in association with reduced sensitivity to CCR5 antagonist TAK-779. Notably, all these substitutions were positioned on the receptors binding surfaces in V1/N2 or V3 loop. In silico structural studies predicted some physical changes of gp120 by substitutions with alterations in viral replication phenotypes. These data suggest that V1/V2 loop is critical for creating a gp120 structure that masks co-receptor binding site compatible with maintenance of viral infectivity, and for tuning a functional balance of gp120 between immune escape ability and infectivity to optimize HIV-1 replication fitness.

Keywords: homology modeling, MD simulation, V1/V2 loop, V3 loop, adaptive mutation

\section{INTRODUCTION}

An envelope glycoprotein (Env) of human immunodeficiency virus type 1 (HIV-1) is synthesized in cells as a precursor gp160, and subsequently cleaved to mature gp120 and gp41. HIV-1 Env works, as a trimer of a gp120-gp41 dimer molecule, on viral entry into target host cells (Freed and Martin, 1995, 2013; Clapham and McKnight, 2002; Wilen et al., 2012). Env-gp120 binds to receptor CD4 and co-receptor CCR5 or CXCR4, whereas Env-gp41 mediates virus-cell membrane fusion. Depending on the co-receptor usage, HIV-1 strains are grouped into CCR5 (R5)-tropic and CXCR4 
(X4)-tropic viruses. During the entry process, viral Env proteins undergo large conformational changes. CD4 induces gp120 conformational change upon their binding, and facilitates subsequent interaction with the co-receptor. Binding of gp120 to the receptor and co-receptor triggers a drastic structural change of gp41, and allows virus-cell membrane fusion. Successful entry is crucial for establishing HIV-1 infection.

HIV-1 Env displays high adaptive capacity. This ability enables HIV-1 to escape from host immune recognition with maintenance of function/structure of Env involved in viral entry. Consequently, HIV-1 Env exhibits an extensive genetic variation. Based on genetic variations, gp 120 consists of discontinuous five conserved regions ( $\mathrm{C} 1$ to $\mathrm{C} 5)$ and five variable regions (V1 to V5; Freed and Martin, 1995). Numerous studies have detailed the role of each region in Env functions. V1/V2 and V3 form flexible loop structures on the gp120 core region (Huang et al., 2005; McLellan et al., 2011) and have been shown to be involved in modulation of the co-receptor usage, cell tropism, replication ability and/or neutralization resistance (Freed and Martin, 1995, 2013; Clapham and McKnight, 2002; Tilton and Doms, 2010; Benjelloun et al., 2012; Wilen et al., 2012; Connell and LortatJacob, 2013; Mascola and Haynes, 2013). Recent crystal and cryo-electron microscopy (cryo-EM) structure studies on Env have revealed that V1/V2 loops are located at the trimer apex in connection with V3 loop, and that the conformational change of V1/V2 loops upon CD4 binding triggers exposure of V3 loop to the co-receptor (Julien et al., 2013; Lyumkis et al., 2013). These results are the indicative of concerted action of V1/V2 and V3 loops during HIV-1 entry and thereby adaptation processes. In this regard, we previously identified adaptive mutations in HIV1 Env-gp120 that are responsible for viral growth enhancement in growth-restrictive cells of an R5-tropic and macaque/human tropic HIV-1 derivative, NL-DT562 (562; Nomaguchi et al., 2008, 2013a; Yamashita et al., 2008). These single-amino acid substitutions were found to be clustered within V1/V2 and V3 domains (Nomaguchi et al., 2013a).

Critical issues that remain to be solved for better understanding of HIV-1 entry and adaptation can be summarized as follows: (1) amino acid residue(s) essential for the Env function are less well-defined; (2) adaptive mutation(s) that augment viral entry efficiency/replication ability are not yet amply described; (3) how the adaptive mutations alter viral phenotypes remain unclear. Although atomic-level information on the natural gp120 structure is essential to clarify each of these issues, such information is still technically hard to obtain. While the small-angle $\mathrm{x}$-ray scattering (SAXS; Guttman et al., 2012) and cryo-EM (Lyumkis et al., 2013) have provided invaluable information on the global fold and shape of gp120 trimer on the virion, these techniques are in principle unable to give the atomic-level information on the protein structure. Meanwhile, the crystal structure analysis is beneficial to obtain the atomic-level information, but most studies have focused on the gp120 core region and are lacking in the flexible loop regions. To date, only a few studies have reported crystal structures of the full-length HIV-1 gp120 trimer (Julien et al., 2013; Pancera et al., 2014; Do Kwon et al., 2015). In those studies, protein preparations for crystallization were bound to anti-gp120 neutralizing antibodies, and/or contained multiple mutations at V3 base/C5 regions for structure stabilization and/or antigenicity modification. These physical modifications might influence structure and function of native gp120. Thus, information on the full-length gp120 structures obtained by distinct approaches would be informative to complement available structural data of the HIV-1 gp120.

In this study, we combined computational and experimental approaches to gain new insights into the roles of gp120 variable loops in HIV-1 entry and their adaptive mutations. By coupling homology modeling and molecular dynamics (MD) simulation, we constructed R5-tropic full-length gp120 models that disclose key structural features to understand gp120 functions: topologies of the variable loops (Guttman et al., 2012; Julien et al., 2013; Lyumkis et al., 2013; Pancera et al., 2014; Do Kwon et al., 2015), conformational masking of V3 tip (Guttman et al., 2012; Munro et al., 2014; Pancera et al., 2014), and CD4-induced conformational rearrangement (Huang et al., 2005; Guttman et al., 2012; Julien et al., 2013; Pancera et al., 2014; Do Kwon et al., 2015). Using the models and $\mathrm{MD}$ simulation, we obtained evidence that V1/V2 loop is critical to create gp120 structure that makes the masking of co-receptor binding site for immune escape compatible with the maintenance of viral infectivity. In parallel, cell-based viral adaptation experiments in the absence of antibodies led to the identification of single amino acid substitutions in Env gp120 for better viral entry and replication. Structural models predicted that these mutations resided in the receptors binding surface and could modulate gp120 structures for receptors binding. Our structural and virological data illustrate a previously less characterized physical function of $\mathrm{V} 1 / \mathrm{V} 2$, i.e., the regulation of V3 conformation, for tuning viral infectivity and immune escape ability to optimize viral replication fitness in given environments.

\section{MATERIALS AND METHODS}

\section{Molecular Modeling of a Full-Length gp120 Monomer in CD4-Free and -Bound States}

Three-dimensional (3-D) models for a full-length gp120 monomer of HIV-1 R5-tropic virus in CD4-free and -bound states were constructed by assembling missing parts of the protein surface onto the gp120 core of JRFL virus (GenBank accession no. U63632), followed by homology modeling. Comparison of gp120 core structures in public database indicated that overall conformations of the cores were similar in the CD4-free and bound states except for V1/V2 stem region. Therefore, we used the core structure with gp41-interactive region of HIV-1 JRFL at a $2.61 \AA$ resolution (PDB code:3JWD; Pancera et al., 2010) as a common platform to construct full-length gp120 models. Because 3JWD represents the core bound to soluble CD4, we replaced its V1/V2 stem region with that of the CD4-free partial core structure (PDB code: 3IDX, a $2.5 \AA$ resolution; Chen et al., 
2009) for modeling of gp120 in the CD4-free state. Other parts of gp120 were same for the present modeling. To supplement missing surface structures of gp120, we used partial structures as follows: V1/V2 (PDB code: 3U4E, a $2.19 \AA$ resolution; McLellan et al., 2011), V3 (PDB code: 2QAD, a $3.3 \AA$ resolution; Huang et al., 2007), V4 (PDB code: 2B4C, a $3.3 \AA$ resolution; Huang et al., 2005), and C5 (PDB code: 1MEQ, solution nuclear magnetic resonance structure; Guilhaudis et al., 2002). The gp120 core and surface structures were used as templates to construct full-length models for JRFL gp120 by homology modeling using 'MOEAlign', a tool for multiple sequence and structure alignment for examining their relatedness ${ }^{1}$, and 'MOE-Homology', a tool to predict structure by the sequence and structure relatedness in the Molecular Operating Environment MOE (Chemical Computing Group Inc.). Obtained models were optimized by energy minimization using MOE and an Amber10: Extended Huckel Theory (EHT) force field implemented in MOE, which combines Amber10 and EHT bonded parameters for the largescale energy minimization (Gerber and Muller, 1995; Case et al., 2005).

\section{Simulation of a Full-Length, Glycosylated gp120 in a CD4-Free State}

A full-length, glycosylated gp120 model in a CD4-free state was subjected to MD simulation essentially as described for simulations of HIV-1/SIV gp120 outer domains (Naganawa et al., 2008; Yokoyama et al., 2012; Kuwata et al., 2013; Yuan et al., 2013). Briefly, a high-mannose oligosaccharide $\mathrm{Man}_{5} \mathrm{GlcNAc}_{2}$ was added to potential $N$-glycosylation sites in gp120 outer domains using Online Glycoprotein Builder ${ }^{2}$. MD simulations were performed by the PMEMD (Particle Mesh Ewald Molecular Dynamics) module in the AMBER 10 program package (Case et al., 2005), employing the AMBER ff99SB-ILDN force field, a protein force field with improved side-chain torsion potentials (Lindorff-Larsen et al., 2010), the GLYCAM06 force field, a biomolecular force field for glycans (Kirschner et al., 2008), and the TIP3P water model for simulations of aqueous solutions (Jorgensen et al., 1983). Bond lengths involving hydrogen were constrained with SHAKE, a constraint algorithm to satisfy a Newtonian motion (Ryckaert et al., 1977), and the time step for all MD simulations was set to 2 fs. A non-bonded cutoff of $10 \AA$ was used. After heating calculations for 20 ps until $310 \mathrm{~K}$ using the NVT ensemble for the constant volume, temperature, and numbers of particles in the system, simulations were executed using the NPT ensemble for the constant pressure, temperature, and numbers of particles in the system at $1 \mathrm{~atm}$, at $310 \mathrm{~K}$, and in $150 \mathrm{mM} \mathrm{NaCl}$ for $50 \mathrm{~ns}$.

\section{Molecular Modeling of HIV-1 gp120 Trimers in CD4-Free and -Bound States}

For modeling of $\mathrm{CD} 4$-free trimer, the $\mathrm{CD} 4$-free monomer model was superposed on the gp120 trimer structure derived from the x-ray crystal structure of Env proteins (PDB code: 4TVP;

${ }^{1}$ https://www.chemcomp.com/journal/align.htm

${ }^{2}$ http://glycam.org/
Pancera et al., 2014). For modeling of CD4-bound trimer, the CD4-bound monomer model was superposed on the gp120 trimer structural image derived from cryo-EM analysis of Env proteins on the virion (PDB code: 3DNO; Liu et al., 2008). Superimpositions of monomer structures were done using the Protein Superpose module in MOE.

\section{Prediction of the Effects of Amino Acid Substitutions on the Stability and Affinity of gp120-CD4 Complex}

The 3-D model of HIV-1 562 full-length gp120 bound to cynomolgus monkey CD4 (GenBank accession no. D63349) was constructed by homology modeling using MOE-Align and MOEHomology, and refined by energy minimization and by the Ramachandran plot using MOE. The full-length gp120 model in a human soluble-CD4 bound state was used as a template for homology modeling. The 562 gp120 and cynomolgus monkey $\mathrm{CD} 4$ complex was used for in silico mutagenesis as described in the study of HIV-1 capsid protein (Nomaguchi et al., 2013b). Changes in the stability and affinity of the gp120-CD4 complex by mutations were computed by using the Protein Design application in MOE, a computational tool for the structure analysis of mutant proteins and for the computational design of protein with desirable properties. Briefly, single-point mutations on the gp120 protein were generated, and ensembles of protein conformations were generated by the LowMode MD module in MOE, a tool of low-mode velocity filtering for conformational search, to calculate average stability and affinity using Boltzmann distribution. Finally, stability and affinity scores of the structures refined by energy minimization were obtained through the scoring function of the Protein Design application.

\section{Simulation of gp120 V3 Loops}

Molecular models for V3 loops of 562 and 562 S304G gp120 proteins were constructed by homology modeling using the x-ray crystal structure of gp120 with V3 loop at a $3.30 \AA$ resolution (PDB code: 2QAD; Huang et al., 2007) as a modeling template. The models represent V3 loop structures of gp120 in the CD4-bound state, because the template gp120 was bound to soluble CD4. The initial V3 models were thermodynamically and physicochemically refined using MOE. MD simulations of V3 loops were done essentially with the same calculation conditions as described above for MD simulations of the full-length gp 120 . After heating calculations for 20 ps until $310 \mathrm{~K}$ using the NVT ensemble, simulations were executed using the NPT ensemble at $1 \mathrm{~atm}$ and at $310 \mathrm{~K}$ for $20 \mathrm{~ns}$. Superimpositions of V3 structures were done using the Protein Superpose module in MOE by coordinating atoms of amino acid residues at $\mathrm{V} 3$ base.

\section{Calculation of Root Mean Square Deviation (RMSD) and Root Mean Square Fluctuation (RMSF) Values}

Root Mean Square Deviation and RMSF values were calculated as previously described to quantify structural dynamics of molecules in the MD simulations (Yokoyama et al., 2012). RMSD values between the heavy atoms of the two superposed proteins 
were used to measure overall structural differences between the two proteins (Case et al., 2005). We also calculated RMSF values of the $\mathrm{C} \alpha$ atoms to obtain information on atomic fluctuations of individual amino acid residues during MD simulations (Case et al., 2005). The 10,000 snapshots obtained from MD simulations of 10-20 ns were used to calculate RMSF values. The average structures during the last $10 \mathrm{~ns}$ of $\mathrm{MD}$ simulations were used as reference structures for RMSF calculation. Both RMSD and RMSF values, which quantify the differences between the average values and those obtained at give times of MD simulations, were calculated using the ptraj module in Amber, a trajectory analysis tool (Case et al., 2005).

\section{Plasmid DNA}

Construction and characterization of various proviral clones designated pNL-DT562, pNL-DT5R, pNL4-3, and pNL-Kp (NL4-3 $\Delta$ Env construct) were previously described (Adachi et al., 1986, 1991; Kamada et al., 2006; Yamashita et al., 2008). Growthenhancing mutations were site-specifically introduced into pNLDT562 by using the QuickChange site-directed mutagenesis kit (Agilent Technologies) as fully described previously (Nomaguchi et al., 2013a, 2014).

\section{Cells}

A human monolayer cell line 293T (Lebkowski et al., 1985) and a cynomolgus macaque lymphocyte cell line HSC-F (Akari et al., 1999) were routinely maintained in Eagle's MEM supplemented with $10 \%$ heat-inactivated fetal bovine serum and in RPMI1640 containing 10\% heat-inactivated fetal bovine serum, respectively.

\section{Transfection, Reverse Transcriptase (RT) Assay, and Infection}

Virus samples were prepared from 293T cells transfected with various proviral clones by the calcium-phosphate coprecipitation method as previously described (Adachi et al., 1986; Kamada et al., 2006; Nomaguchi et al., 2013a,b, 2014). Virion-associated RT activity was measured to determine virus amounts as described previously (Willey et al., 1988; Nomaguchi et al., 2013b). For determination of viral growth kinetics, equal amounts (RT units) of virus preparations were inoculated into HSC-F cells, and infected cells were cultured in the presence of IL-2 $(50 \mathrm{U} / \mathrm{ml})$. Virus replication was monitored every 3 days by RT activity in the culture supernatants.

\section{Entry Assay}

Input virus samples for entry assays were prepared from transfected $293 \mathrm{~T}$ cells as above, and quantified by the HIV1 p24 antigen enzyme-linked immunosorbent assay (ELISA) kit (ZeptoMetrix Corporation). Entry assays were performed as previously described (von Schwedler et al., 1993; Adachi et al., 1996). Briefly, HSC-F cells $\left(10^{6}\right)$ were incubated with viruses (100 ng of p24) for $2 \mathrm{~h}$ at $4^{\circ} \mathrm{C}$, extensively washed, and collected as viral binding fractions. To determine virus entry level, HSCF cells treated with viruses at $4^{\circ} \mathrm{C}$ as above were trypsinized for $5 \mathrm{~min}$ at $37^{\circ} \mathrm{C}$, extensively washed, and incubated for $2 \mathrm{~h}$ at $37^{\circ} \mathrm{C}$ for preparation of viral entry fractions. The binding and entry fractions were lysed, and the p24 level of samples was determined by the ELISA kit as above. Entry efficiency of each sample was calculated as p24 level of entry fraction/p24 level of binding fraction. NL4-3 and NL-Kp (NL4-3 $\Delta$ Env) were used as positive and negative controls, respectively.

\section{Inhibitor Sensitivity Assays}

To determine the co-receptor usage, HSC-F cells $\left(10^{5}\right)$ were treated with/without $1 \mu \mathrm{M}$ co-receptor antagonists (CXCR4 antagonist AMD3100 and CCR5 antagonist TAK-779) at $37^{\circ} \mathrm{C}$ for $1 \mathrm{~h}$, and equal virus amounts ( $10^{5} \mathrm{RT}$ units) were inoculated into the pretreated cells. Co-receptor antagonists were present in the cultures throughout the experiment. Virus replication was monitored by RT activity released into the culture supernatants. Viral yields in test cultures relative to those on the peak day in cultures without antagonists were calculated. X4-tropic HIV1 derivative 5R served as a control. To determine sensitivity of 562 and its gp120-mutants to TAK-779, HSC-F cells $\left(10^{5}\right)$ were treated with serial 10 -fold dilutions of TAK-779 $\left(1-10^{-5} \mu \mathrm{M}\right)$ at $37^{\circ} \mathrm{C}$ for $1 \mathrm{~h}$, and viruses $\left(10^{6} \mathrm{RT}\right.$ units for 562 and $10^{5} \mathrm{RT}$ units for gp120-mutants) were then inoculated into the pretreated cells. TAK-779 concentration was maintained in the cultures throughout the experiment. Virus replication was monitored by RT activity released into the culture supernatants. Viral yields in test cultures relative to those on the peak day in cultures without TAK-779 were calculated.

\section{RESULTS}

\section{Simulation of a Full-Length, Glycosylated gp120 Protein Predicts a Thermodynamically Stable Arrangement of V1/V2 and V3 Loops in the CD4-Free State}

Because full-length gp120 structures of the same strain in CD4free and -bound states were unavailable at atomic levels in the public database, we constructed them with the aid of in silico science (see Materials and Methods for details of modeling). Unliganded gp120 protein of HIV-1 is characterized to exist as semi-stable state, i.e., local minimum of energy state, through interactions of variable loops on the core surface (Kwon et al., 2012). Maintaining such unique conformation is considered to be critical for generating its functions in virus entry into target cells and in immune/drug escape (Kwon et al., 2012). To identify the functional arrangement of the variable loops at semi-stable state of unliganded gp120 protein, we performed MD simulation for the CD4-free gp120 model (Figure 1A). To mimic physical properties of the native gp120, we added high-mannose oligosaccharide $\mathrm{Man}_{5} \mathrm{GlcNAc}_{2}$ at the potential $\mathrm{N}$-glycosylation sites in gp120 outer domains of the initial model before MD simulation. RMSD between the initial structure and structures at given times of simulation sharply increased in the beginning and reached a near plateau after 20 ns of the MD simulations (Figure 1B). These results suggest that the structural 

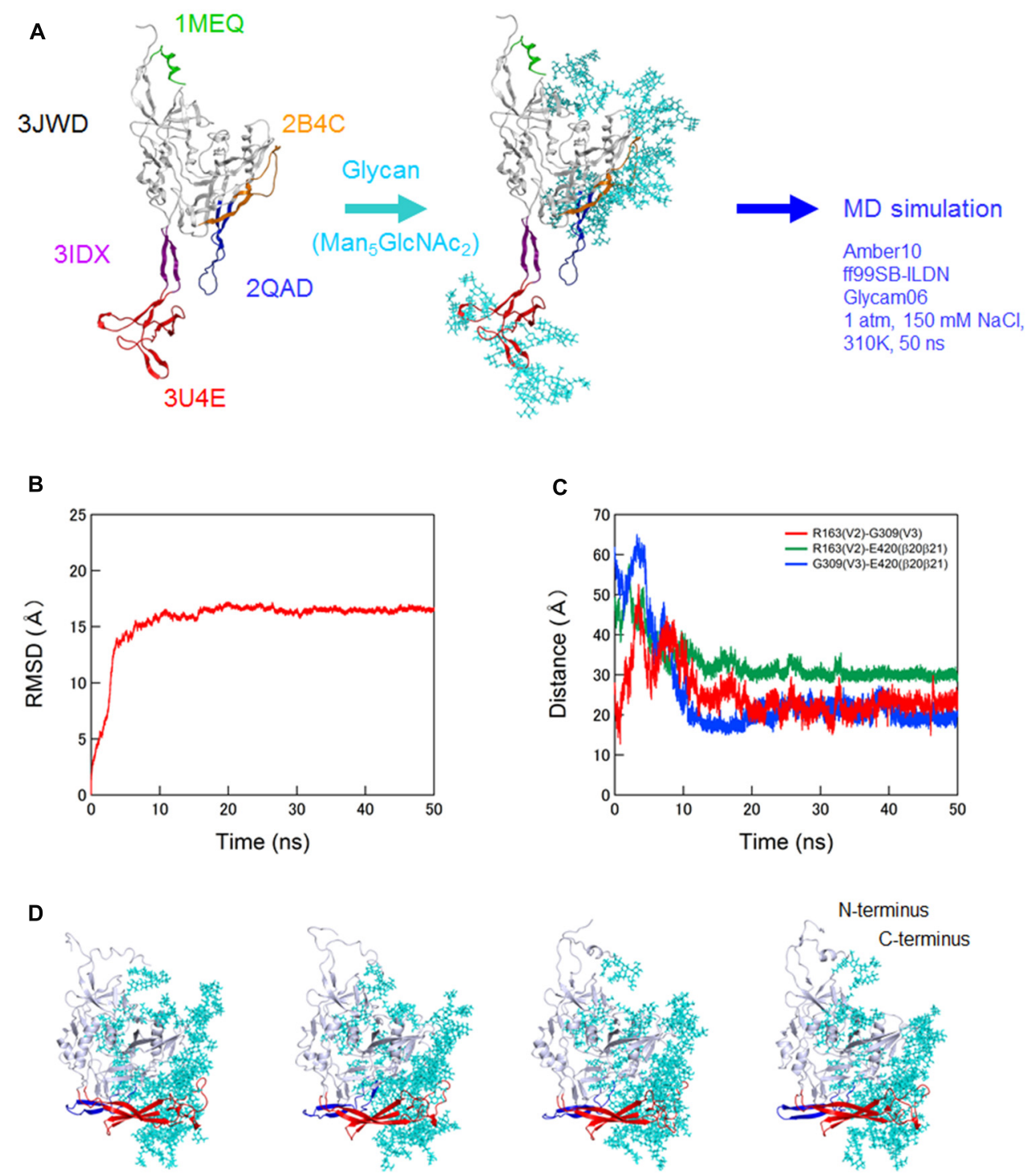

$20 \mathrm{~ns}$

$30 \mathrm{~ns}$

$40 \mathrm{~ns}$

$50 \mathrm{~ns}$

E

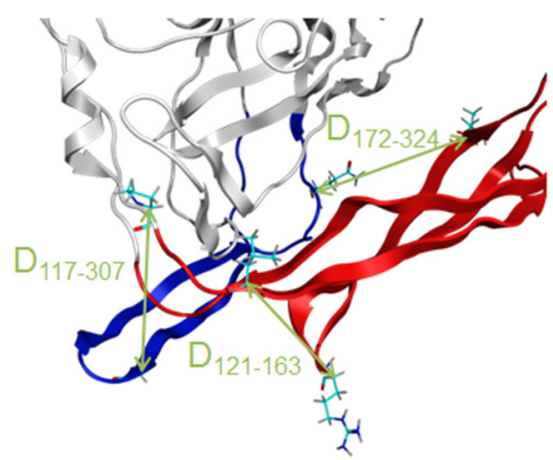

\begin{tabular}{|c|c|c|c|}
\hline & \multicolumn{2}{|c|}{ Distances (Â) } & \multirow[b]{2}{*}{ Differences ( $\AA$} \\
\hline & MD model & $\begin{array}{c}\text { Crystal } \\
\text { structure }\end{array}$ & \\
\hline$D_{117-307}$ & 18.30 & 15.57 & 2.73 \\
\hline$D_{121-163}$ & 14.41 & 16.22 & 1.81 \\
\hline $\mathrm{D}_{172-324}$ & 17.14 & 14.82 & 2.32 \\
\hline
\end{tabular}




\begin{abstract}
FIGURE 1 | Continued
Molecular dynamics (MD) simulation of a full-length, glycosylated HIV-1JRFL gp120 monomer. (A) Schematic representation of molecular modeling and MD simulation. Molecular model for a full-length, glycosylated gp120 monomer of HIV-1 R5-tropic virus JRFL in a CD4-free state was constructed by assembling of HIV-1 gp120 parts and homology modeling. PDB codes of the structures used for modeling are indicated on the left. Thermodynamically and physically refined model was subjected to MD simulation using the PMEMD module in the AMBER 10 program package as described for simulations of HIV-1/SIV gp120 outer domains (Naganawa et al., 2008; Yokoyama et al., 2012; Kuwata et al., 2013; Yuan et al., 2013). (B) Time course of RMSD between the initial model and models at given times of MD simulation. RMSD values were calculated with trajectories at every 2 fs of MD simulation using the ptraj module in Amber 10 . (C) Time course of distances between residues in V2 and V3 (red line), residues in V2 and $\beta 20-\beta 21$ (green line), and residues in V3 and $\beta 20-\beta 21$ (blue line) in gp120. (D) Structures of a full-length, glycosylated gp120 monomer at 20, 30, 40, and 50 ns of MD simulations. V1/N2 and V3 regions are highlighted by red and blue colors, respectively. (E) Distances between the variable loops and core. The distances between the $\mathrm{C} \alpha$ of P117 at core neighboring $\mathrm{V} 1$ base and $\mathrm{C} \alpha$ of $\mathrm{G} 307$ at the tip of $\mathrm{V} 3$ loop $\left(D_{117-307}\right), C \alpha$ of $L 121$ at core neighboring V1 base and the $C \alpha$ of R163 at V2 loop ( $\left.D_{121-163}\right)$, and $C \alpha$ of $L 172$ at V2 loop and the C $\alpha$ of $Q 324$ at V3 base $\left(D_{172}-324\right)$ were calculated with the gp120 model at $50 \mathrm{~ns}$ simulation and $\mathrm{x}$-ray crystal structure (4TVP) to quantitatively compare relative $3-\mathrm{D}$ locations of the $\mathrm{V} 1 \mathrm{~N} 2$ and V3 loops on the core. Amino acid numbers are based on those of the JRFL Env protein (GenBank accession no. U63632).
\end{abstract}

distortions were relieved shortly after the start of simulation under thermodynamic driving forces in solution. The data also predict that the glycosylated gp120 structure can reach a state of thermodynamic equilibrium in solution.

Of note, distances between V2 and V3, V2 and $\beta 20-\beta 21$, and $V 3$ and $\beta 20-\beta 21$ in gp120 reached a near plateau with low levels of fluctuations (Figure 1C). The data indicate that a stable arrangement of V1/V2 and V3 on the glycosylated gp120 core exists in solution in the CD4-free state. The data also suggest that V1/V2 and V3 interactions confine their free movements. The results are consistent with a previous report on the physical function of V1/V2 loop for the regulation of gp120 conformation (Kwon et al., 2012). In this MD-predicted equilibrium state, $\mathrm{V} 1 / \mathrm{V} 2$ and V3 loops positioned at the opposite side from the $\mathrm{N}$ - and C-terminal regions and continually fluctuated over time (Figures 1C,D). V1/V2 was extended from inner to outer domains along core, whereas V3 was extended from outer to inner domains along core (Figure 1D). The relative 3-D locations of V1/V2 loop, V3 loop, and N/C termini on the core in the equilibrium state agree with those reported in the structural model constructed with SAXS data of a CD4-free full-length gp120 (Guttman et al., 2012). The relative 3-D locations also agree with those in the cryo-electron tomography reconstruction model (Lyumkis et al., 2013) and the x-ray crystal structures (Julien et al., 2013; Pancera et al., 2014; Do Kwon et al., 2015) for gp140 containing whole gp120 and the ectodomain of gp41. To quantitatively address this issue, we measured the distances between the variable loops and core at three sites $\left(\mathrm{D}_{117-307}, \mathrm{D}_{121-163}\right.$, and $\left.\mathrm{D}_{172-324}\right)$ using our gp120 model at 50 ns simulation and $\mathrm{x}$-ray crystal structure at pre-fusion state (4TVP; Figure 1E). The differences in distances between the model and x-ray crystal structure were $2.73,1.81$, and $2.32 \AA$ for the $\mathrm{D}_{117-307}, \mathrm{D}_{121-163}$, and $\mathrm{D}_{172-324}$, respectively, which were within the range of resolution of the $4 \operatorname{TVP}(3.1 \AA)$. Thus, the gp120 structure model at $50 \mathrm{~ns}$ in the equilibrium state closely recapitulates relative $3-\mathrm{D}$ locations of $\mathrm{V} 1 / \mathrm{V} 2$ and $\mathrm{V} 3$ loops on the core of the x-ray crystal structure.

\section{Conformational Masking of a Co-Receptor Binding Site in the Ligand-Free, Trimeric gp120}

Env gp120 V3 loop element is critical for co-receptor binding for HIV-1 entry into target cells and determines viral co-receptor tropism (Hwang et al., 1991). The tip region of V3 loop therefore constitutes a potent neutralization epitope (Goudsmit et al., 1988; Palker et al., 1988; Rusche et al., 1988; Javaherian et al., 1989). However, studies on viral neutralization (Bou-Habib et al., 1994; Stamatatos and Cheng-Mayer, 1995; Krachmarov et al., 2005; Lusso et al., 2005), gp120 conformational dynamics (Munro et al., 2014), and V3 sequence evolution (Sato et al., 1999; Shiino et al., 2000) consistently indicate that this epitope is generally concealed from the antibody access in the primary isolates. HIV-1 R5tropic virus clone JRFL used in the present modeling study is also a neutralization-resistant tier- 2 primary isolate from an HIV1 infected individual (Koyanagi et al., 1987). To assess whether our model here predicts this conformational masking on virion, we constructed a full-length gp120 trimer model by superposing the structure at $50 \mathrm{~ns}$ of MD simulation on the x-ray crystal structure of envelope gp140 trimer (PDB code: 4TVP; Figure 2). Whereas V3 tip was exposed on the gp120 at the monomeric state (Figure 2A), this region was positioned toward the center at the trimeric state so that V3 tip is sterically concealed (Figure 2B). These results are consistent with those in the SAXS-based gp120 model (Guttman et al., 2012), the x-ray crystal structure of gp140 (Pancera et al., 2014), and the single-molecule fluorescence resonance energy transfer imaging (Munro et al., 2014).

\section{Conformational Rearrangement of V1/V2 and V3 Loops After CD4 Binding}

Virological (Moore et al., 1992; Lee et al., 1997) and structural (Huang et al., 2005; Julien et al., 2013; Lyumkis et al., 2013; Pancera et al., 2014; Do Kwon et al., 2015) studies consistently suggest that following CD4 binding, the gp120 surface undergoes conformational changes from co-receptor-binding-incompetent to -competent form. To address this issue, we constructed a full-length gp120 model in a CD4-bound state (Figure 3A). The global shape and structural topologies of variable loops of this model are consistent with those in the SAXS-based gp120 model in the CD4-bound state (Guttman et al., 2012). Comparison of our gp120 models in the CD4-free (Figure 2A) and -bound (Figure 3A) states shows that the core structures of the two models are similar except for the secondary structure of $\mathrm{V} 1 / \mathrm{V} 2$ stem. This difference, however, caused marked difference in arrangements of V1/V2 and V3 loops on the core. In the CD4-free state, V1/V2 was positioned near V3 loop so that the variable surfaces could interact with each other to maintain a 


\section{A}

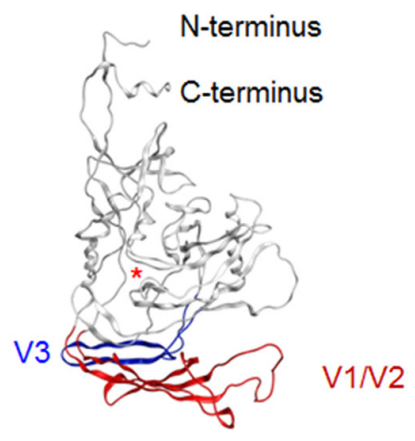

B
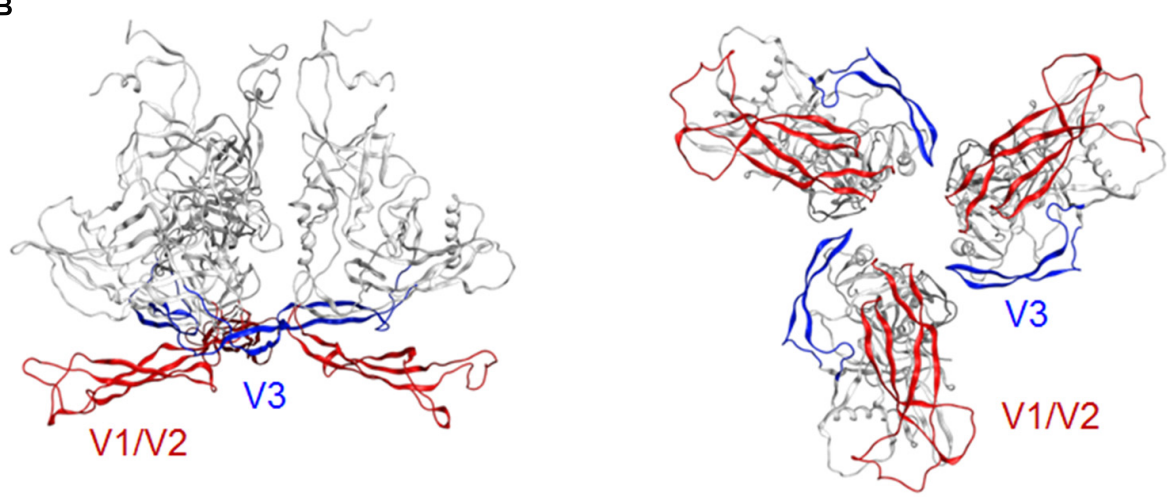

FIGURE 2 | Model for a full-length HIV-1JRFL gp120 in a CD4-free state. (A) Full-length gp120 monomer model. The structure at 50 ns of MD simulation in Figure 1D is shown. Glycans are removed for clear view of the outer domain. A red asterisk indicates the location of CD4-binding loop. (B) Full-length gp120 trimer model. The model was constructed by superposing CD4-free monomer model on the x-ray crystal structure of Env gp140 protein (PDB code: 4TVP; Pancera et al., 2014). Side and top views are shown on the left and on the right, respectively. V1N2 and V3 regions are highlighted by red and blue colors, respectively.

stable arrangement on the core (Figures $\mathbf{1 B}-\mathbf{D}$, and $\mathbf{2 A}$ ). In the CD4-bound state, however, V1/V2 loop is rearranged near the bound CD4 to support CD4 binding: V1/V2 stem formed bridging sheet in combination with $\beta 20-\beta 21$ loop so that V1/V2 loop could interact with CD4 instead of V3 (Figure 3A). This $\mathrm{V} 1 / \mathrm{V} 2$ repositioning is consistent with the SAXS-based gp120 model for the CD4-bound state (Guttman et al., 2012). Due to this conformational rearrangement in the CD4-bound state, V3 loop became free from structural constraints imposed by the $\mathrm{V} 1 / \mathrm{V} 2$ interactions and protruded out of the core surface for coreceptor interactions in the trimer state (Figure 3B). These results agree with what was proposed previously for the conformational rearrangements of gp120 variable surface upon CD4 binding (Guttman et al., 2012).

\section{Each Individual Adaptive Single-Amino Acid Mutation in V1/V2 and V3 Regions of 562 Env-gp120 Enhances Viral Growth Potential}

Our structural study suggests that V1/V2 region is critical for determining viral phenotypes via regulating topology and conformation of V3 loop. Considering that V1/V2 and V3 loop are variable, we assumed that these regions might be critical for adaptive mutations under changing environments.
To address this issue, we examined here how HIV-1 adapts in our cell-based HIV-1 assay system (Kamada et al., 2006; Nomaguchi et al., 2008, 2013a). While multiple selective factors including the host immune response drive virus adaptation/evolution in individuals, only those that affect viral replication ability itself contribute to virus adaptation in the cell culture system, providing simple experimental means to study HIV-1 adaptation for entry. Previously, we performed continuous cultivation of 562 and its X4tropic version NL-DT5R (5R) under growth-restrictive conditions imposed by macaque cell lines (Nomaguchi et al., 2013a). In our repeated experiments, 562 and 5R frequently acquired growth-enhancing adaptive mutations in both Polintegrase and Env-gp120 regions (Nomaguchi et al., 2013a, 2014).

In this study, we focused on analyzing the changes in the 562 Env-gp120 to understand structural bases for growth adaptation. Remarkably, the nine single-amino acid substitutions frequently emerged during the adaptation experiments were all positioned at V1/V2 or V3 domain (Nomaguchi et al., 2013a; Figure 4A). Authentic amino acid residues at the mutation sites are relatively well-conserved among HIV-1 strains, and represented the most dominant ones (Figure 4B). Substituted amino acid residues by the adaptations were all found in nature, but usually at much lower frequencies as 


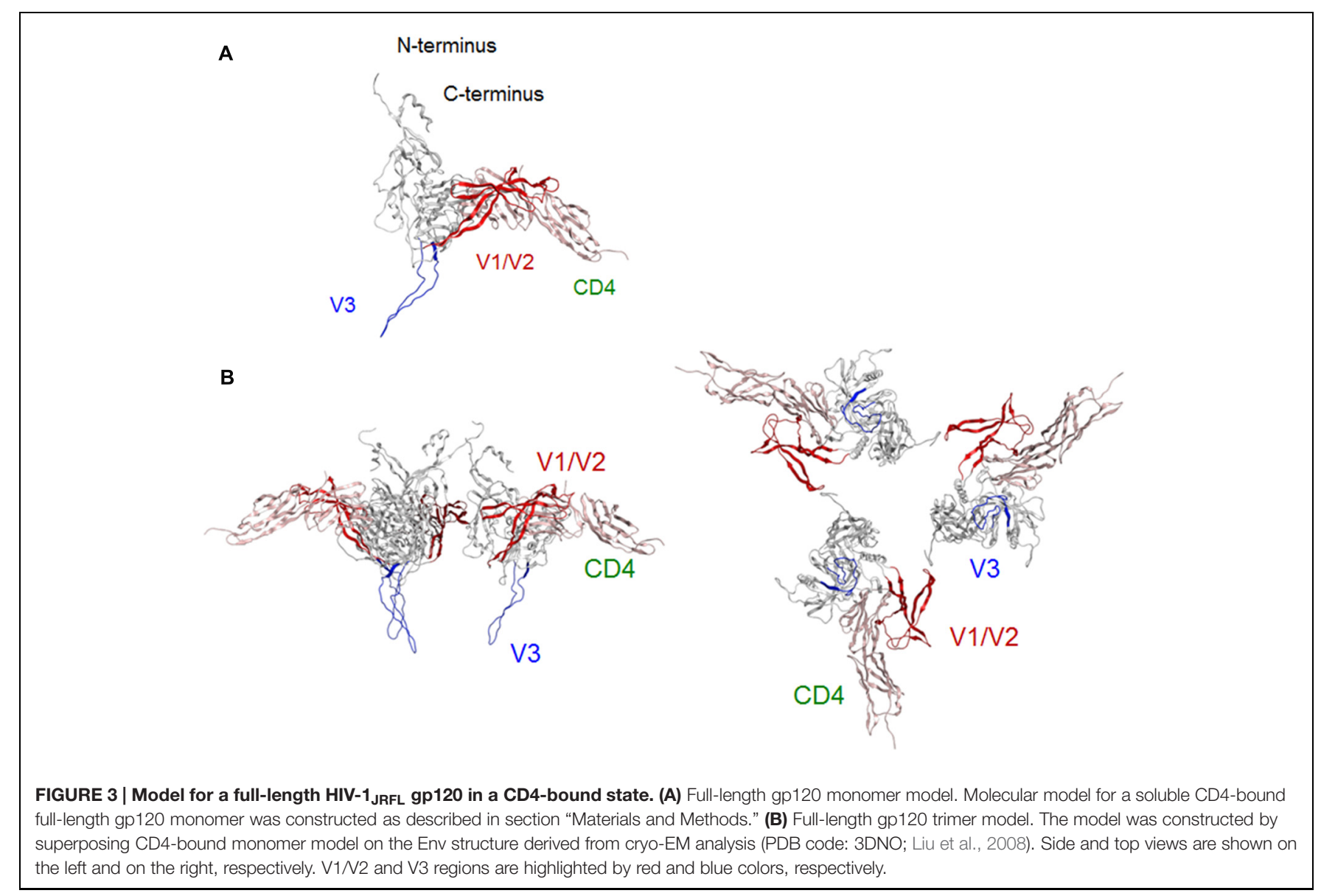

compared with the authentic ones (Figure 4B). These data suggest that the nine sites are intrinsically variable in nature, yet receive constraints on changes under standard replication and transmission conditions. To address biological impacts of these single substitutions, we comparatively analyzed the growth-potentials of a parental clone 562 and its mutants carrying each amino acid substitution. Seven substitutions, L124F, N132K, G150R, F174L, S304G, I307V, and G310R, markedly enhanced replication ability relative to that of 562 (Figure 5A). Furthermore, these mutations except for G310R similarly promoted viral growth in human cells (data not shown). The effect of P181T and G308E substitutions on viral growth was modest (Figure 5B), and therefore, these substitutions were not included in the subsequent analyses. To examine whether a combination of substitutions has an additive enhancing effect, we constructed proviral double mutant clones containing various combinations of substitutions, and compared their replication abilities with those of parental 562 and single mutant viruses (Figure 5C). Although viruses carrying combined substitutions in V1/V2 and V3 grew more efficiently than 562, additive enhancing effects resulted from these double substitutions and a double V3 substitution (S304G and I307V) were not observed. Similar results were obtained for the other double substitutions of V1/V2 and V3 loops (data not shown).

\section{Adaptive Mutations in 562 Env-gp120 Contribute to Increase in Entry Efficiency via the Enhancement of Affinity for CCR5}

Next, we examined the effect of these substitutions on viral entry efficiency. Virus samples were prepared from 293T cells transfected with proviral constructs (562 and 562 mutants carrying each growth-enhancing substitution). Entry assays in HSC-F cells were then performed in a two-step reaction: virus binding step in which virus-cell mixtures were kept at $4^{\circ} \mathrm{C}$; subsequent virus penetration step in which cells after virus binding were incubated at $37^{\circ} \mathrm{C}$. Entry efficiency of each virus sample was calculated as p24 level of penetrated virus into cells relative to that of attached virus onto cells. Notably, all the seven substitutions enhanced the viral entry efficiency in cells (Figure 6A). These data suggest that adaptive mutations in 562 Env-gp120 promote viral replication by increasing entry efficiency.

Since it has been shown that alterations in V1/V2 and V3 regions modulate the co-receptor usage (Freed and Martin, 1995, 2013; Clapham and McKnight, 2002; Tilton and Doms, 2010; Benjelloun et al., 2012; Wilen et al., 2012; Connell and Lortat-Jacob, 2013; Mascola and Haynes, 2013), we determined the susceptibility of above mutants to co-receptor antagonists. 
A

\begin{tabular}{|c|c|c|c|}
\hline & 120 & $\begin{array}{ccc}\text { L124F } & \text { N132K } & \text { G150R } \\
\downarrow & \downarrow & \downarrow \\
\text { KLTPICVTLHCTILKNATNTKSSN-WKEMDRGEIKNC }\end{array}$ & 155 \\
\hline & 120 & . . . . . N.KDVNATNT.ND.E--GT.E. . . . . & 154 \\
\hline & 121 & .......S.K..D...D...N. .SGRMI.EK..... & 157 \\
\hline
\end{tabular}

V2 $562 \quad 156$ JRFL 155

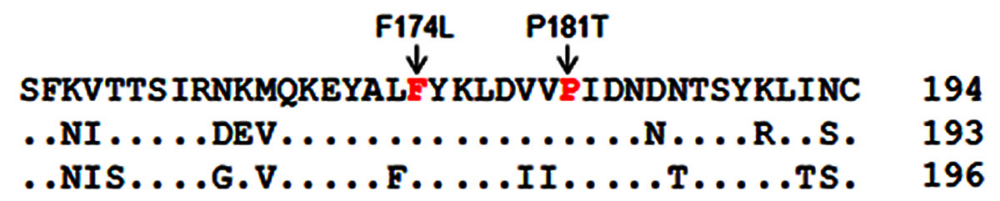
HXB2 158

154

157

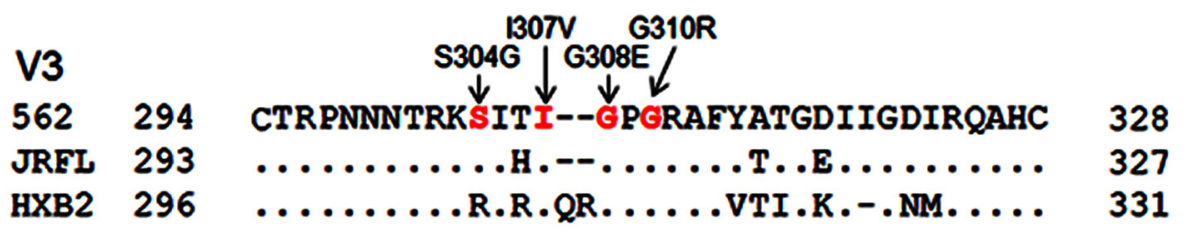

B

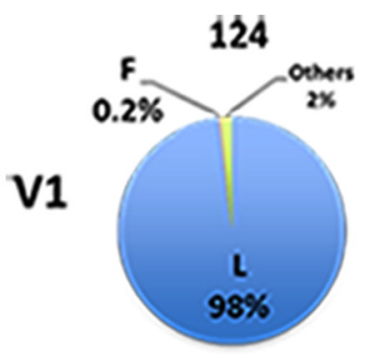

174

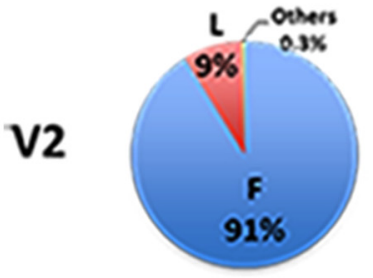

304

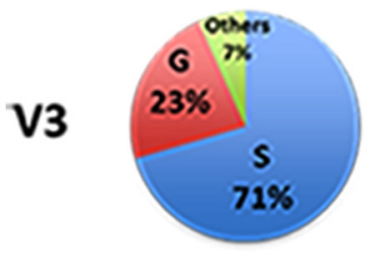

132

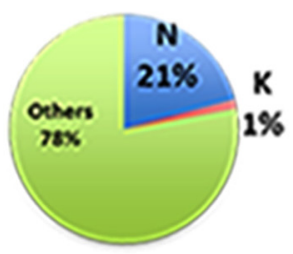

181
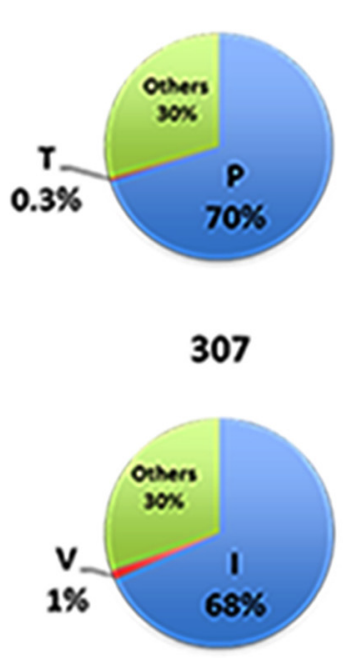

150

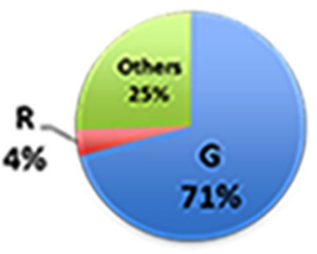

$4 \%$

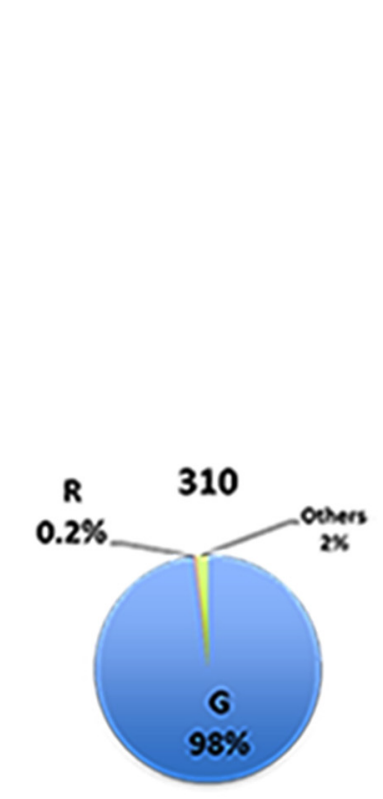




\section{FIGURE 4 | Continued}

Genetic information on HIV-1 gp120 adaptive mutations in the present study. (A) Location of HIV-1 adaptive mutations in EnV-gp120. Amino acid sequences in V1 to V3 regions of HIV-1 R5-tropic 562 virus (Nomaguchi et al., 2013a) carrying the SF162 env gene (GenBank accession no. EU123924; Kawamura et al., 1994) are aligned with those of R5-tropic JRFL clone (GenBank accession no. U63632) and of X4-tropic HXB2 clone (GenBank accession no. K03455). Assignment of V1, V2, and V3 regions is based on gp120 structure: V1N2 (PDB code: 3U4E), V3 (PDB code: 2QAD). (B) Frequency of authentic (light blue) and replaced (red) amino acid residues. Others (green) represent amino acid residues other than authentic and replaced ones. Naturally occurring amino acid residues at specific positions, where adaptive 562-gp120 mutations are located, were investigated in an HIV-1 subtype B population from different geographic regions in the world (19,419 sequences), and are graphically shown. The 19,419 sequences were obtained from the HIV Sequence Database (http://www.hiv.lanl.gov/content/sequence/HIV/ mainpage.html).

A

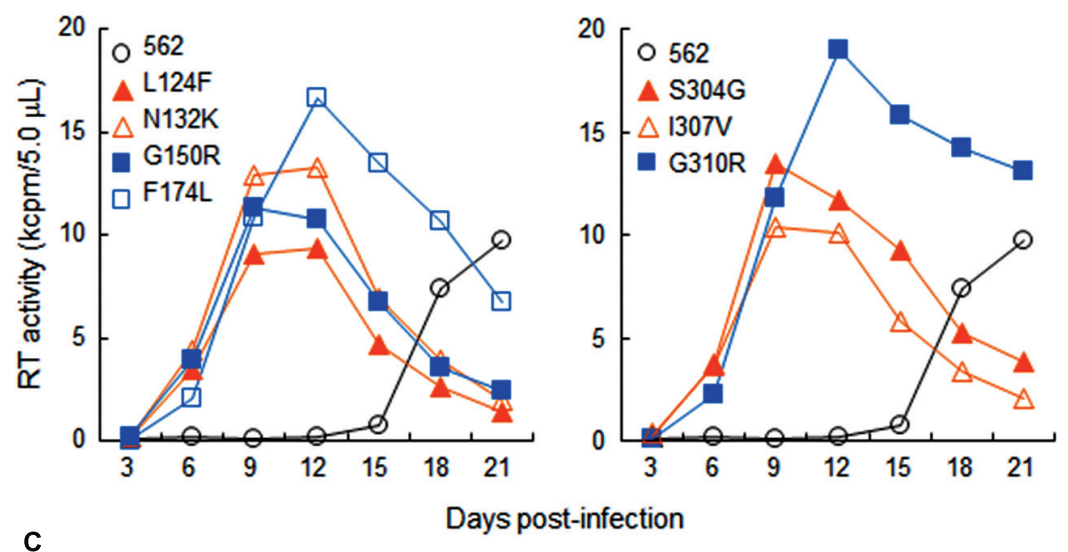

B

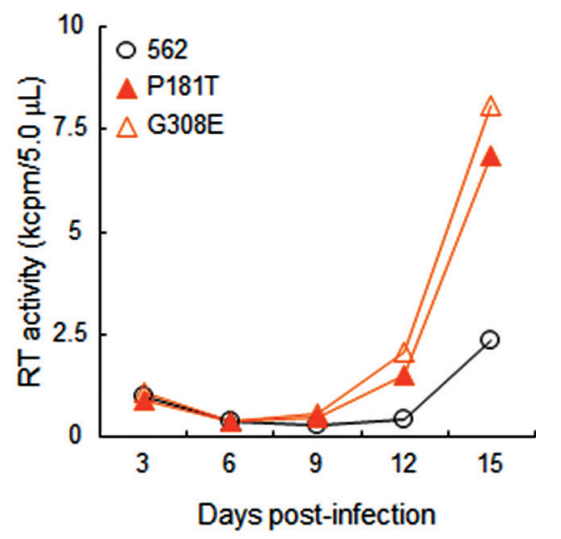

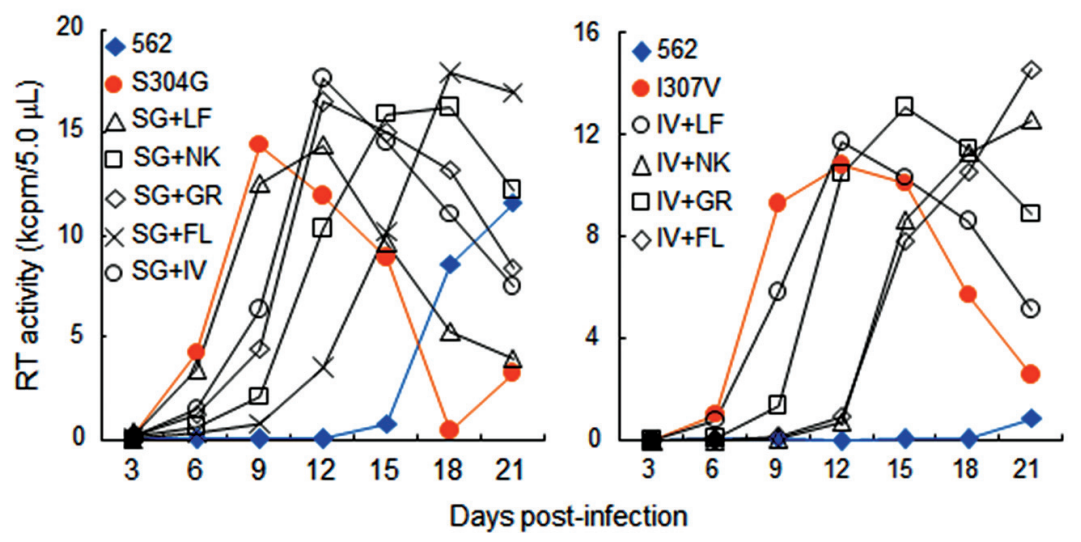

FIGURE 5 | Growth properties of 562 derivative viruses carrying various adaptive mutations in gp120. (A) Growth kinetics of variant viruses carrying a single adaptive mutation. Virus samples were prepared from 293T cells transfected with indicated proviral clones, and inoculated into HSC-F cells. Virus replication was monitored by RT activity released into the culture supernatants. Data of left and right panels were obtained from the same experiment, and the same result for 562 is separately shown as a control. Infection condition: $2 \times 10^{5}$ RT units/2 $\times 10^{5}$ cells. (B) Growth properties of slowly growing variant viruses carrying a single adaptive mutation. Experiment was performed as described above. Infection condition: $4 \times 10^{6} \mathrm{RT}$ units/10 ${ }^{6}$ cells. (C) Growth kinetics of variant viruses carrying two adaptive mutations. Experiments were performed as above. LF, L124F; NK, N132K; GR, G150R; FL, F174L; SG, S304G; IV, I307V; GR, G310R. Infection conditions: left, $2 \times 10^{5} \mathrm{RT}$ units $/ 2 \times 10^{5}$ cells; right, $5 \times 10^{4} \mathrm{RT}$ units $/ 1 \times 10^{5}$ cells.

Virus samples were prepared from $293 \mathrm{~T}$ cells transfected with proviral constructs (5R, 562 and 562 mutants carrying each growth-enhancing substitution), and inoculated into HSC-F cells pretreated with the antagonists. As is clear in Figure 6B, the replication of 5R was inhibited in the presence of AMD3100 (anti-CXCR4), but not TAK-779 (anti-CCR5). Conversely, the replication of 562 and its single-amino acid mutants (L124F, N132K, G150R, F174L, S304G, I307V, and G310R) was restricted in the presence of TAK-779, but not AMD3100 (Figure 6B). These results indicate that the viral co-receptor tropism is not affected at all by the above mutations in Env-gp120.

It has been demonstrated that the Env with enhanced affinity for co-receptor displays increased resistance to entry inhibitors (e.g., T-20) and co-receptor antagonists (e.g., TAK779; Reeves et al., 2002; Lobritz et al., 2007; Pacheco et al., 
A

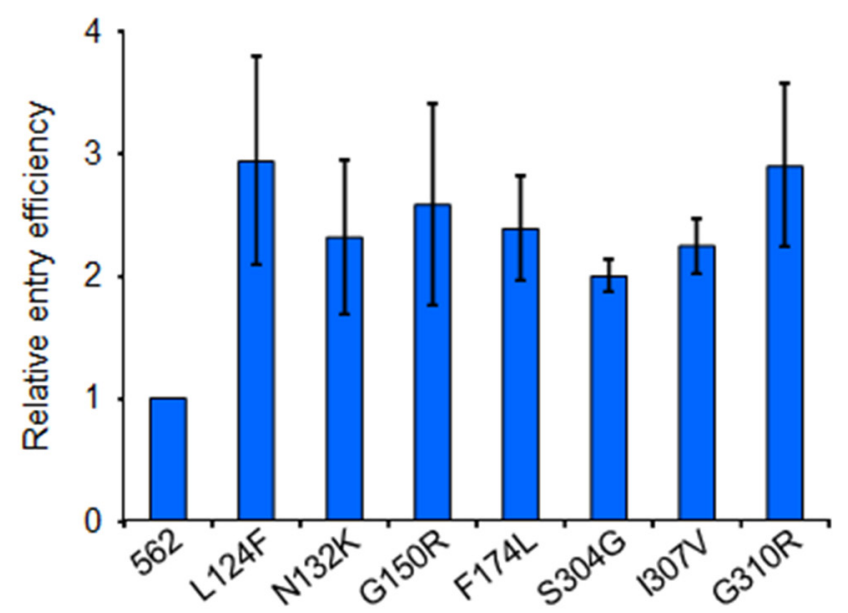

B

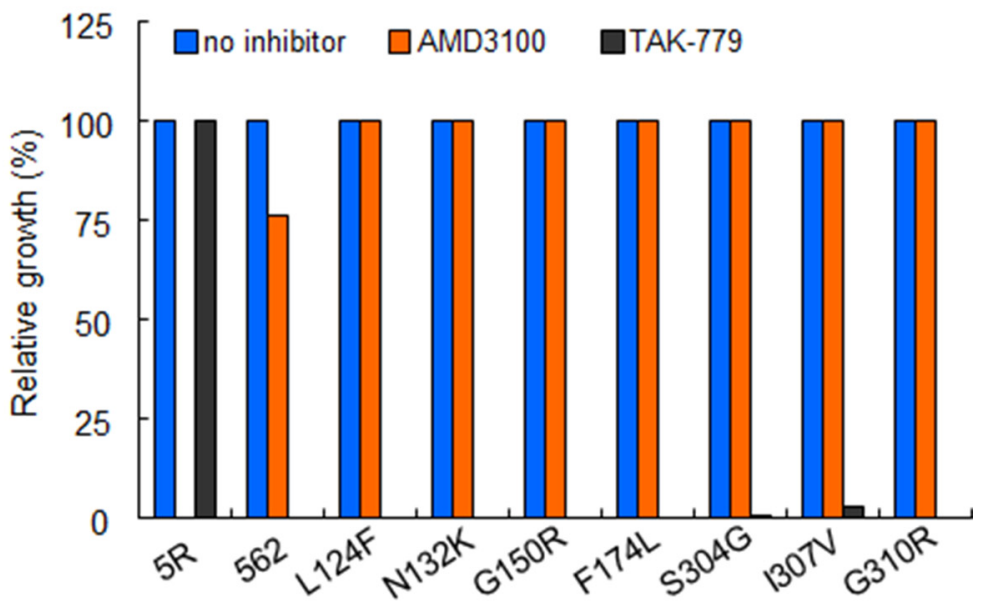

C

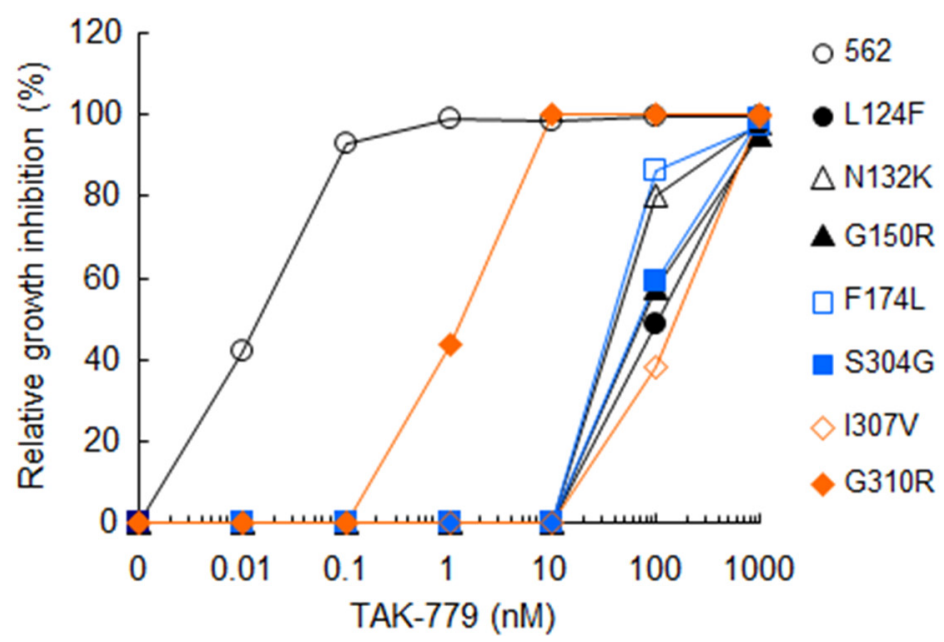




\section{FIGURE 6 | Continued}

Effects of growth-enhancing mutations in gp120 on viral entry. (A) Entry efficiency into HSC-F cells of 562 and 562 carrying indicated single substitutions. Virus samples were prepared as in Figure 5, and entry assays were performed as described in section "Materials and Methods." Values obtained for $\Delta$ Env construct (NL-Kp) were subtracted from those for test samples. Entry efficiency of each virus relative to that of 562 is presented. (B) Co-receptor usage of various viruses. Infection of HSC-F cells with viruses was performed as described above, and infected cells were cultured in the absence or presence (1 $\mu \mathrm{M})$ of antagonists (CXCR4 antagonist AMD3100 or CCR5 antagonist TAK-779). Virus replication was monitored by RT activity released into the culture supernatants. Viral yields in test cultures relative to those on the peak day in cultures without antagonists were determined. $5 R$ and 562 served as controls. (C) Sensitivity of 562 and its mutants to TAK-779. Virus samples prepared as above were inoculated into HSC-F cells pretreated with the indicated concentration of TAK-779. Virus replication was monitored by RT activity released into the culture supernatants. Viral yields in test cultures relative to those on the peak day in cultures without TAK-779 were determined and presented as \% inhibition. Representative results from three independent experiments are shown.

2008). Therefore, we compared the sensitivity of 562 and its mutants to TAK-779 (Figure 6C). HSC-F cells pretreated with increasing concentrations of TAK-779 were infected with 562 or its mutants (L124F, N132K, G150R, F174L, S304G, I307V, or G310R). A high concentration of TAK-779 was required to completely restrict the replication of viral clones containing mutations L124F/N132K/G150R/F174L/S304G/I307V, and a mutant clone carrying G310R was modestly more resistant to TAK-779 relative to 562 . These results show that the growthenhancing mutations in 562 Env-gp120 clearly enhance, without exception, the affinity for CCR5. Collectively, the results obtained show that adaptive mutations in 562 Env-gp120 promote viral replication by enhancing entry efficiency through increase in the affinity for CCR5.

\section{Three-D Locations and Structural Impacts of the Adaptive Mutations}

In parallel with the above experimental study, we investigated 3-D locations and possible structural impacts of the adaptive mutations to gain insights into how the mutations change the viral phenotype. Interestingly, whereas the seven substitutions were sporadically distributed along the gp120 core surface before CD4 binding (Figure 7A), all of them were rearranged at or near the potential sites for CD4 or co-receptor binding after CD4 binding (Figure 7B): they were positioned either around the $\mathrm{CD} 4$ binding surface in the V1/V2 region or at the V3 tip/stem region. The data suggest that some of these mutations may influence the gp120 structure for receptors binding.

To address the influence on gp120 binding to CD4, we constructed a 3-D model of 562 full-length gp120 bound to cynomolgus monkey CD4 (GenBank accession no. D63349), and performed in silico mutagenesis as described in the study of HIV-1 capsid protein (Nomaguchi et al., 2013b). Four point mutations (N132K in V1/F174L in V2/S304G in $\mathrm{V} 3 / \mathrm{I} 307 \mathrm{~V}$ in $\mathrm{V} 3$ ) were predicted to reduce the stability of gp120-CD4 complex, whereas the other three (L124F in $\mathrm{V} 1 / \mathrm{G} 150 \mathrm{R}$ in $\mathrm{V} 1 / \mathrm{G} 310 \mathrm{R}$ in V3) were expected to increase the stability (Figure 8A). Thus, some adaptive mutations were assumed to be disadvantageous in terms of the stability of CD4 bound structure. Nevertheless, they appeared reproducibly during adaptation in macaque cells (Nomaguchi et al., 2013a), indicating that some advantage(s) surpasses the harmful effect. Affinity scores predicted that the point mutations L124F and
G150R in V1, which increase the stability of gp120-CD4 complex (Figure 8A), also increase the binding affinity of gp120 and CD4 (Figure 8B). These results suggest that these V1 mutations can cause structural changes in gp120 to improve CD4 binding.

V3 loop conformation is critical for interactions with the N-terminal portion of CCR5 (Huang et al., 2005, 2007), and amino acid substitutions within V3 loop can influence its conformational dynamics, changing the viral co-receptor tropism and neutralization sensitivity (Naganawa et al., 2008; Yokoyama et al., 2012; Kuwata et al., 2013). We therefore examined whether V3 adaptive substitutions affect the conformational dynamics of V3 loop using MD simulation (Figure 9). We selected S304G substitution in this study, because the substitution most reproducibly and frequently emerged in our adaptation experiments (Nomaguchi et al., 2013a). Introduction of S304G substitution into the V3 stem of 562 clone resulted in enhancement of the RMSD fluctuations (Figure 9A) and in rearrangement of the V3 tip (Figure 9B). This substitution exchanges serine with glycine, the smallest amino acid residue, and rationally increased magnitudes of the V3 loop fluctuation (Figure 9C), leading to reduced probability to form a stable antiparallel $\beta$-sheet that contains 5-6 mainchain hydrogen bonds (Figure 9D, left panel) and a turn at base/stem region of V3 during the simulations (Figure 9D, right panel). These data suggest that S304G substitution can modulate structural features of V3 loop for co-receptor interactions.

\section{DISCUSSION}

In this study, we combined computational and experimental approaches to address how HIV-1 Env gp120 mechanically modulates viral phenotypes and contributes to viral adaptation. By coupling homology modeling and $\mathrm{MD}$ simulation, we constructed full-length gp120 models that disclose key features on the gp120 structure, i.e., topologies of the variable loops (Guttman et al., 2012; Julien et al., 2013; Lyumkis et al., 2013; Pancera et al., 2014; Do Kwon et al., 2015), conformational masking of V3 tip (Guttman et al., 2012; Munro et al., 2014; Pancera et al., 2014), and CD4-induced conformational rearrangement (Huang et al., 2005; Guttman et al., 2012; Julien et al., 2013; Pancera et al., 2014; Do Kwon et al., 2015; Figures 1-3). In parallel, cell-based adaptation experiments led to the identification of single amino acid substitutions 

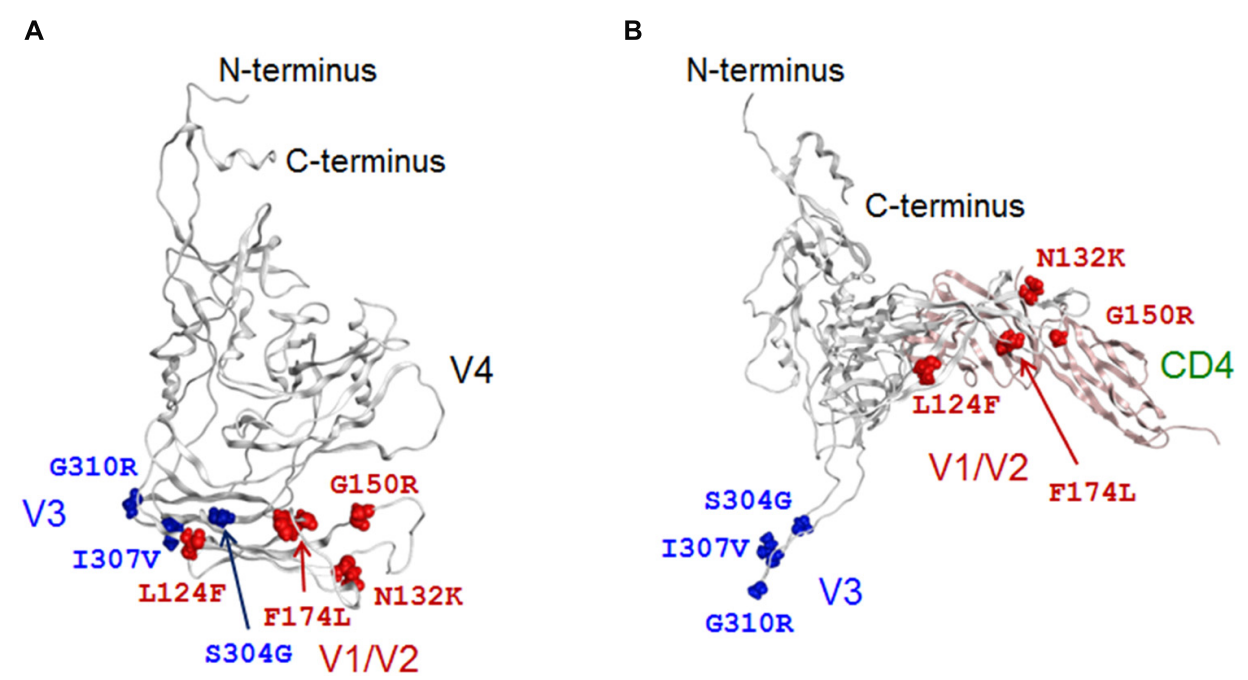

FIGURE 7 | The 3-D locations in a full-length gp120 of adaptive mutations. Adaptive amino acid substitutions are highlighted by colored globules on the gp120 models in CD4-free (A) and CD4-bound (B) states. Amino acid residues in V1N2 and V3 regions are highlighted by red and blue colors, respectively. For details of the two models, see Figures $2 \mathbf{A}$ and $\mathbf{3 A}$.

in Env gp120 for enhanced viral entry/growth through better affinity for CCR5 (Figures 4-6). Our structural models predicted that these mutations resided on the receptor binding surfaces and could modulate gp120 structures for receptors binding (Figures 7-9). These findings suggest that amino acid substitutions on the receptors binding surface are a key mechanism to tune viral entry efficiency during HIV-1 growth adaptation.

Most importantly, MD simulation of the full-length gp120 molecule revealed a physical function of V1/V2 loop. In the CD4-free state, V1/V2 loop, which initially positioned away from V3 loop, settled into a fixed position near the V3 loop to fit in a thermodynamically stable arrangement (Figure 1). The results suggest presence of semi-stable state, i.e., local minimum of energy state for the full-length unliganded gp120. In this semi-stable state, V1/V2 and V3 loops were stably arranged near the core under mutually interactive compact positions (Figures 1B-D). This stable arrangement of variable loops on the core is reasonable from the viewpoint of protein chemistry, because unliganded protein generally fold into a compact conformation with minimum protein surface in solution. More importantly, the relative $3-\mathrm{D}$ locations of the variable loops on the core surface in the semi-stable state agree with those reported in the previous experimental studies (Guttman et al., 2012; Julien et al., 2013; Lyumkis et al., 2013; Pancera et al., 2014; Do Kwon et al., 2015) and explain the well-known experimental observation, i.e., conformational masking of V3 neutralization epitope in the trimeric context (Guttman et al., 2012; Munro et al., 2014; Pancera et al., 2014) with keeping CD4 binding site accessible (Figure 2). Thus, the present unliganded gp120 model in the semi-stable state recapitulates structural and virological features of the functional gp120 revealed by various experimental methods. Our MD simulation study additionally suggests that V1/V2 loop has an intrinsic physicochemical feature that attracts
V3 loop in the CD4-free state. These findings are well consistent with a previous notion that in the CD4-free state, shift to CD4bound conformation is restrained by interactions of V1/V2 and V3 loops (Kwon et al., 2012). In the CD4-bound state, however, V1/V2 loop repositioned near the bound CD4 to support CD4 binding, while V3 loop formed exposed conformation for co-receptor binding via relief from V1/V2 loop (Figure 3). Taken together, these results strongly suggest that V1/V2 loop functions as a nano device to create a gp120 structure that masks co-receptor binding site for immune evasion compatible with maintenance of viral infectivity.

Present study also provides structural bases to understand how V1/V2 and V3 loops mechanically modulate various biological phenotypes of HIV-1. Ligand-free gp120 models (Figures 1 and 2) predict that changes in the physicochemical features of V1/V2 or V3 loop, by amino acid substitutions or glycosylation, would alter the nature of attractive interactions among these loops in the CD4-free state. Such changes in turn could cause changes in the exposure levels of co-receptor binding site on virions and thereby would alter viral neutralization sensitivity to anti-V3 antibodies (Etemad-Moghadam et al., 1999; Pinter et al., 2004; Krachmarov et al., 2006; Naganawa et al., 2008), as well as fusogenic activity of gp120 and viral infectivity (Sullivan et al., 1993; Ogert et al., 2001; Laird et al., 2008; Cavrois et al., 2014). In addition, CD4-bound gp120 models (Figure 3), in silico mutagenesis of gp120 (Figure 8), and MD simulation of V3 loop (Figure 9) suggest that changes in the loops could alter the efficiency of receptors binding in the CD4-bound state. For example, L124F and G150R substitutions in V1 loop were predicted to increase gp120 binding affinity for CD4 (Figure 8), and S304G substitution in V3 loop was expected to alter conformational dynamics of V3 loop (Figure 9). Although structural impacts by the adaptive mutations remained characterized further, present findings suggest that a combination(s) of multiple rather than 


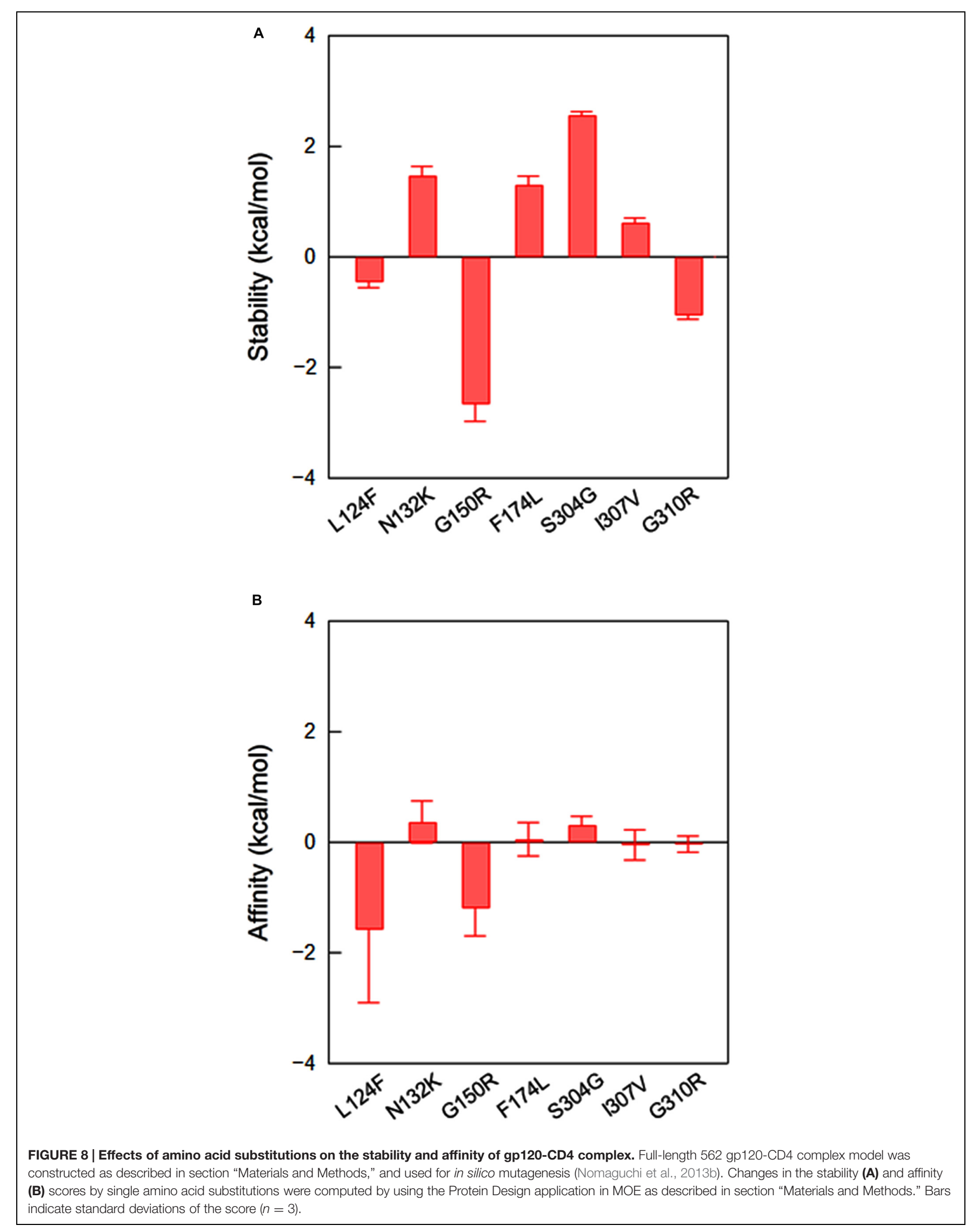




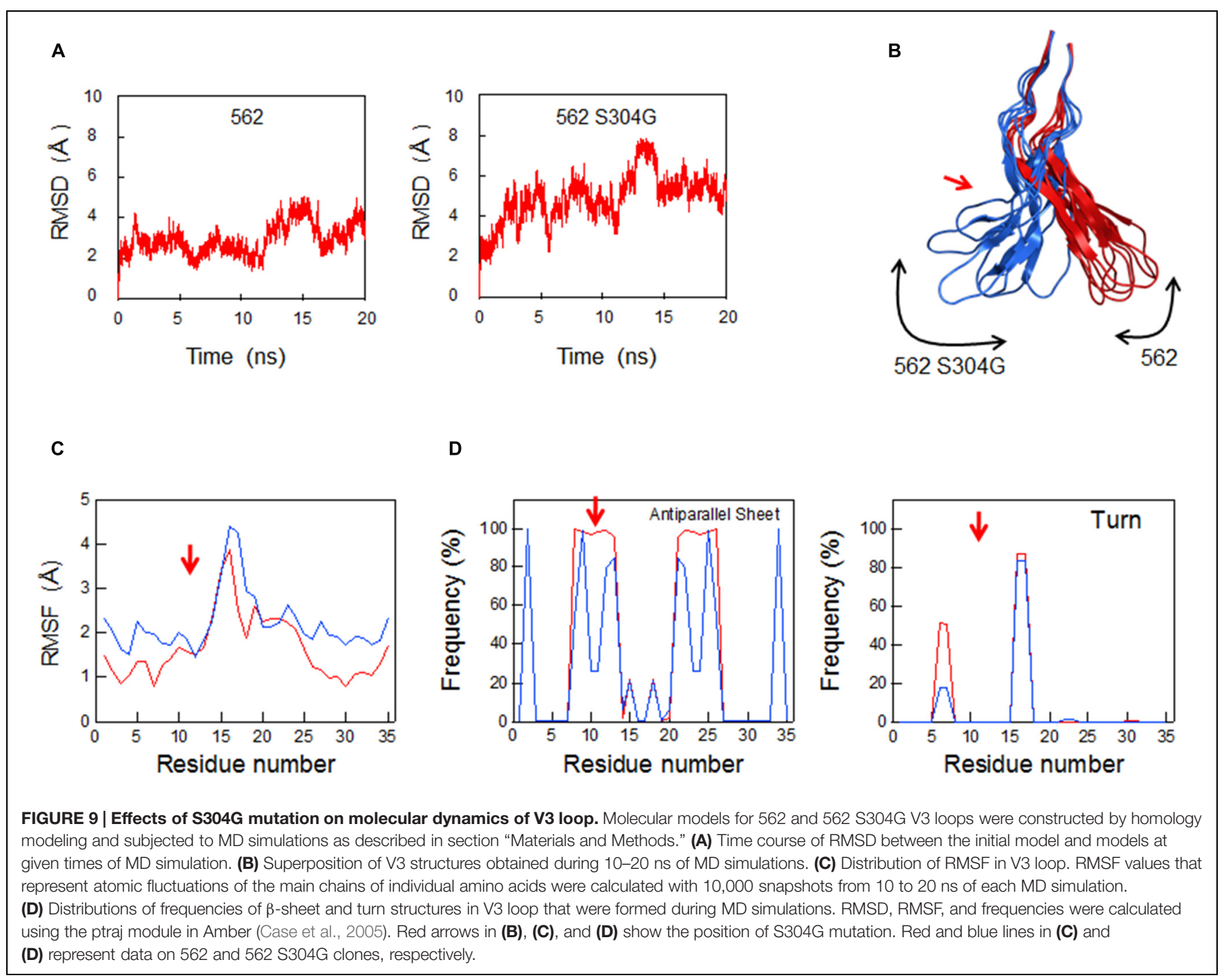

a single mechanism, such as alterations in physical interactions among V1/V2 and V3 loops in the unliganded state and/or alterations in physical interactions with CD4/CCR5, governed the modulation of growth phenotypes of Env-gp120 mutants in this study.

Present study further provides structural insights into the HIV-1 adaptation. Considering that V3 tip constitutes a potent neutralization epitope (Goudsmit et al., 1988; Palker et al., 1988; Rusche et al., 1988; Javaherian et al., 1989), HIV-1 needs to keep evading swarms of newly generated anti-V3-tip antibodies against replicating viruses during persistent infection in infected individuals. A key evasion mechanism is considered to be masking of the co-receptor binding site in V3 (Bou-Habib et al., 1994; Stamatatos and Cheng-Mayer, 1995; Krachmarov et al., 2005; Lusso et al., 2005), i.e., V3 tip. Meanwhile, persisting viruses also should maintain their infectivity that requires the exposure of the co-receptor binding site (Huang et al., 2005), i.e., V3 tip. As suggested in the present study, V1/V2 loop seems to be an essential device to create structural compatibility of gp120 for these reciprocal requirements. Considering that V1/V2 and V3 loops are intrinsically highly variable (HIV Sequence Database ${ }^{3}$ ), it is conceivable that configurations of V1/V2 and V3 loops would also have variations in nature. The structural variations on the gp120 surface generated by variable loops would in turn allow natural selection of the fittest viruses as suggested in previous (Nomaguchi et al., 2013a) and present (Figures 5-9) studies on the HIV-1 Env adaptation. Thus, our structural and virological data are consistent with the assumption that variable V1/V2 and V3 loops are key devices to create structural variations of the gp120 surface to optimize replication fitness under given environments.

The authentic amino acid residues, at the sites that acquired adaptive mutations are located, are relatively well-conserved among virus strains from all HIV-1 subtypes (Zolla-Pazner and Cardozo, 2010; for subtype B, see Figure 4B). Interestingly, both $\mathrm{F}(\mathrm{Phe}) / \mathrm{L}(\mathrm{Leu})$ at position 174 and both $\mathrm{S}(\mathrm{Ser}) / \mathrm{G}(\mathrm{Gly})$ at position 304 exist as natural variants (Figure 4B). Mutation of

${ }^{3}$ http://www.hiv.lanl.gov/content/sequence/HIV/mainpage.html 
F174 residue has been shown to affect binding of a V2-specific antibody 697-D and of neutralizing antibodies PG9 and PG16 (Zolla-Pazner and Cardozo, 2010). It is intriguing to see how the replication of viruses carrying $\mathrm{F} 174 \mathrm{~L}$ mutation is regulated in individuals. Notably, a particular V3 mutation (S304G) reproducibly appeared with high frequency in the long-term cultures of 562-infected macaque cell lines, and enhanced viral replication ability not only in macaque cells but also in human cells (Nomaguchi et al., 2013a). Even for primary HIV-1 isolates from infected patients, serine and glycine at this position coexist within an individual patient in some cases (Troyer et al., 2005; Delobel et al., 2007; Recordon-Pinson et al., 2013). These results suggest that the $\mathrm{S} 304 \mathrm{G}$ mutation is a very powerful mutation to increase replication fitness of R5-tropic HIV-1 virus in primate cells. Nevertheless, this mutation has been reported to be minor in an R5-tropic virus population in humans (serine, $88 \%$ and glycine, 12\%; Hung et al., 1999) and in SHIV SF162P3-infected macaques (Harouse et al., 2001; Ho et al., 2007; Tasca et al., 2008). These findings suggest the presence of some selective pressure(s) that restrict overgrowth of the S304G variant in vivo. In this regard, we previously reported that V3 mutation is very potent in modulating fluctuation and conformation of the interaction surface of gp120 outer domain (Naganawa et al., 2008; Yokoyama et al., 2012). Therefore, the S304G mutation may augment efficiency of gp120 binding not only to co-receptors but also to neutralization antibodies. Further study is necessary to address this issue.

In summary, our in silico analysis revealed the impact of HIV1 Env-gp120 MD on viral replication. We show here structural and virological evidences that V1/V2 and V3 loops of HIV-1 Env gp120 function as key nano devices to create the Env structure suitable for immune escape compatible with maintenance of viral infectivity, or to acquire adaptive mutations for enhancing viral replication ability. Interlinking in silico science with virology is a powerful strategy for better understanding of HIV-1 adaptation and its biological significance.

\section{REFERENCES}

Adachi, A., Gendelman, H. E., Koenig, S., Folks, T., Willey, R., Rabson, A., et al. (1986). Production of acquired immunodeficiency syndrome-associated retrovirus in human and nonhuman cells transfected with an infectious molecular clone. J. Virol. 59, 284-291.

Adachi, A., Kawamura, M., Tokunaga, K., and Sakai, H. (1996). "Methods for HIV/SIV gene analysis," in Viral Genome Methods, ed. K. W. Adolph (Boca Raton, FL: CRC Press), 43-53.

Adachi, A., Ono, N., Sakai, H., Ogawa, K., Shibata, R., Kiyomasu, T., et al. (1991). Generation and characterization of the human immunodeficiency virus type 1 mutants. Arch. Virol. 117, 45-58. doi: 10.1007/BF01310491

Akari, H., Fukumori, T., Iida, S., and Adachi, A. (1999). Induction of apoptosis in Herpesvirus saimiri-immortalized T lymphocytes by blocking interaction of CD28 with CD80/CD86. Biochem. Biophys. Res. Commun. 263, 352-356. doi: 10.1006/bbrc.1999.1364

Benjelloun, F., Lawrence, P., Verrier, B., Genin, C., and Paul, S. (2012). Role of human immunodeficiency virus type 1 envelope structure in the induction of broadly neutralizing antibodies. J. Virol. 86, 13152-13163. doi: 10.1128/JVI.01110-12

Bou-Habib, D. C., Roderiquez, G., Oravecz, T., Berman, P. W., Lusso, P., and Norcross, M. A. (1994). Cryptic nature of envelope V3 region epitopes protects

\section{AUTHOR CONTRIBUTIONS}

MY: design of the work; acquisition, analysis, and interpretation of data for the work; drafting the work; final approval of the manuscript; agreement to be accountable for all aspects of the work. MN: design of the work; acquisition, analysis, and interpretation of data for the work; drafting the work; final approval of the manuscript; agreement to be accountable for all aspects of the work. ND: acquisition and analysis of data for the work; revising the work; final approval of the manuscript; agreement to be accountable for all aspects of the work. TK: design of the work; revising the work; final approval of the manuscript; agreement to be accountable for all aspects of the work. AA: design of the work; interpretation of data for the work; drafting the work; final approval of the manuscript; agreement to be accountable for all aspects of the work. HS: design of the work; interpretation of data for the work; drafting the work; final approval of the manuscript; agreement to be accountable for all aspects of the work.

\section{FUNDING}

This study is mainly supported by: a Grant-in-Aid for Scientific Research (B) (26293104) to AA and a Grant-in Aid for Scientific Research on Innovative Areas (25115519) to HS from the Japan Society for the Promotion of Science; grants to HS (Research on HIV/AIDS project no. H22-003) and to MY (Research on HIV/AIDS project no. H25-006) from the Ministry of Health, Labour and Welfare of Japan.

\section{ACKNOWLEDGMENT}

We thank Ms. Kazuko Yoshida for editorial assistance.

primary monocytotropic human immunodeficiency virus type 1 from antibody neutralization. J. Virol. 68, 6006-6013.

Case, D. A., Cheatham, T. E. III, Darden, T., Gohlke, H., Luo, R., Merz, K. M. Jr., et al. (2005). The Amber biomolecular simulation programs. J. Comput. Chem. 26, 1668-1688. doi: 10.1002/jcc.20290

Cavrois, M., Neidleman, J., Santiago, M. L., Derdeyn, C. A., Hunter, E., and Greene, W. C. (2014). Enhanced fusion and virion incorporation for HIV-1 subtype C envelope glycoproteins with compact V1/V2 domains. J. Virol. 88, 2083-2094. doi: 10.1128/JVI.02308-13

Chen, L., Kwon, Y. D., Zhou, T., Wu, X., O’Dell, S., Cavacini, L., et al. (2009). Structural basis of immune evasion at the site of CD4 attachment on HIV-1 gp120. Science 326, 1123-1127. doi: 10.1126/science.11 75868

Clapham, P. R., and McKnight, A. (2002). Cell surface receptors, virus entry and tropism of primate lentiviruses. J. Gen. Virol. 83, 1809-1829. doi: 10.1099/00221317-83-8-1809

Connell, B. J., and Lortat-Jacob, H. (2013). Human immunodeficiency virus and heparan sulfate: from attachment to entry inhibition. Front. Immunol. 4:385. doi: 10.3389/fimmu.2013.00385

Delobel, P., Nugeyre, M. T., Cazabat, M., Pasquier, C., Marchou, B., Massip, P., et al. (2007). Population-based sequencing of the V3 region of env for predicting the coreceptor usage of human immunodeficiency virus 
type 1 quasispecies. J. Clin. Microbiol. 45, 1572-1580. doi: 10.1128/JCM. 02090-06

Do Kwon, Y., Pancera, M., Acharya, P., Georgiev, I. S., Crooks, E. T., Gorman, J., et al. (2015). Crystal structure, conformational fixation and entry-related interactions of mature ligand-free HIV-1 Env. Nat. Struct. Mol. Biol. 22, 522531. doi: $10.1038 /$ nsmb.3051

Etemad-Moghadam, B., Sun, Y., Nicholson, E. K., Karlsson, G. B., Schenten, D., and Sodroski, J. (1999). Determinants of neutralization resistance in the envelope glycoproteins of a simian-human immunodeficiency virus passaged in vivo. J. Virol. 73, 8873-8879.

Freed, E. O., and Martin, M. A. (1995). The role of human immunodeficiency virus type 1 envelope glycoproteins in virus infection. J. Biol. Chem. 270, 23883-23886. doi: 10.1074/jbc.270.41.23883

Freed, E. O., and Martin, M. A. (2013). "Human immunodeficiency viruses: replication," in Fields Virology, eds D. M. Knipe and P. M. Howley (Philadelphia, PA: Lippincott Williams \& Wilkins), 1502-1560.

Gerber, P. R., and Muller, K. (1995). MAB, a generally applicable molecular force field for structure modelling in medicinal chemistry. J. Comput. Aided Mol. Des. 9, 251-268. doi: 10.1007/BF00124456

Goudsmit, J., Debouck, C., Meloen, R. H., Smit, L., Bakker, M., Asher, D. M., et al. (1988). Human immunodeficiency virus type 1 neutralization epitope with conserved architecture elicits early type-specific antibodies in experimentally infected chimpanzees. Proc. Natl. Acad. Sci. U.S.A. 85, 4478-4482. doi: 10.1073/pnas.85.12.4478

Guilhaudis, L., Jacobs, A., and Caffrey, M. (2002). Solution structure of the HIV gp120 C5 domain. Eur. J. Biochem. 269, 4860-4867. doi: 10.1046/j.14321033.2002.03187.x

Guttman, M., Kahn, M., Garcia, N. K., Hu, S. L., and Lee, K. K. (2012). Solution structure, conformational dynamics, and CD4-induced activation in full-length, glycosylated, monomeric HIV gp120. J. Virol. 86, 8750-8764. doi: 10.1128/JVI.07224-11

Harouse, J. M., Gettie, A., Eshetu, T., Tan, R. C., Bohm, R., Blanchard, J., et al. (2001). Mucosal transmission and induction of simian AIDS by CCR5-specific simian/human immunodeficiency virus SHIV(SF162P3). J. Virol. 75, 19901995. doi: 10.1128/JVI.75.4.1990-1995.2001

Ho, S. H., Tasca, S., Shek, L., Li, A., Gettie, A., Blanchard, J., et al. (2007). Coreceptor switch in R5-tropic simian/human immunodeficiency virus-infected macaques. J. Virol. 81, 8621-8633. doi: 10.1128/JVI.00759-07

Huang, C. C., Lam, S. N., Acharya, P., Tang, M., Xiang, S. H., Hussan, S. S., et al. (2007). Structures of the CCR5 N terminus and of a tyrosinesulfated antibody with HIV-1 gp120 and CD4. Science 317, 1930-1934. doi: 10.1126/science. 1145373

Huang, C. C., Tang, M., Zhang, M. Y., Majeed, S., Montabana, E., Stanfield, R. L., et al. (2005). Structure of a V3-containing HIV-1 gp120 core. Science 310, 1025-1028. doi: $10.1126 /$ science. 1118398

Hung, C. S., Vander Heyden, N., and Ratner, L. (1999). Analysis of the critical domain in the V3 loop of human immunodeficiency virus type 1 gp 120 involved in CCR5 utilization. J. Virol. 73, 8216-8226.

Hwang, S. S., Boyle, T. J., Lyerly, H. K., and Cullen, B. R. (1991). Identification of the envelope V3 loop as the primary determinant of cell tropism in HIV-1. Science 253, 71-74. doi: 10.1126/science.1905842

Javaherian, K., Langlois, A. J., McDanal, C., Ross, K. L., Eckler, L. I., Jellis, C. L., et al. (1989). Principal neutralizing domain of the human immunodeficiency virus type 1 envelope protein. Proc. Natl. Acad. Sci. U.S.A. 86, 6768-6772. doi: 10.1073/pnas.86.17.6768

Jorgensen, W. L., Chandrasekhar, J., Madura, J. D., Impey, R. W., and Klein, M. L. (1983). Comparison of simple potential functions for simulating liquid water. J. Chem. Phys. 79, 926-935. doi: 10.1063/1.445869

Julien, J. P., Cupo, A., Sok, D., Stanfield, R. L., Lyumkis, D., Deller, M. C., et al. (2013). Crystal structure of a soluble cleaved HIV-1 envelope trimer. Science 342, 1477-1483. doi: 10.1126/science. 1245625

Kamada, K., Igarashi, T., Martin, M. A., Khamsri, B., Hatcho, K., Yamashita, T., et al. (2006). Generation of HIV-1 derivatives that productively infect macaque monkey lymphoid cells. Proc. Natl. Acad. Sci. U.S.A. 103, 16959-16964. doi: 10.1073/pnas.0608289103

Kawamura, M., Ishizaki, T., Ishimoto, A., Shioda, T., Kitamura, T., and Adachi, A. (1994). Growth ability of human immunodeficiency virus type 1 auxiliary gene mutants in primary blood macrophage cultures. J. Gen. Virol. 75, 2427-2431. doi: 10.1099/0022-1317-75-9-2427

Kirschner, K. N., Yongye, A. B., Tschampel, S. M., Gonzalez-Outeirino, J., Daniels, C. R., Foley, B. L., et al. (2008). GLYCAM06: a generalizable biomolecular force field. Carbohydrates. J. Comput. Chem. 29, 622-655. doi: 10.1002/jcc.20820

Koyanagi, Y., Miles, S., Mitsuyasu, R. T., Merrill, J. E., Vinters, H. V., and Chen, I. S. (1987). Dual infection of the central nervous system by AIDS viruses with distinct cellular tropisms. Science 236, 819-822. doi: 10.1126/science.3646751

Krachmarov, C. P., Honnen, W. J., Kayman, S. C., Gorny, M. K., Zolla-Pazner, S., and Pinter, A. (2006). Factors determining the breadth and potency of neutralization by V3-specific human monoclonal antibodies derived from subjects infected with clade A or clade B strains of human immunodeficiency virus type 1. J. Virol. 80, 7127-7135. doi: 10.1128/JVI.02619-05

Krachmarov, C., Pinter, A., Honnen, W. J., Gorny, M. K., Nyambi, P. N., Zolla-Pazner, S., et al. (2005). Antibodies that are cross-reactive for human immunodeficiency virus type 1 clade a and clade B v3 domains are common in patient sera from Cameroon, but their neutralization activity is usually restricted by epitope masking. J. Virol. 79, 780-790. doi: 10.1128/JVI.79.2.780790.2005

Kuwata, T., Takaki, K., Yoshimura, K., Enomoto, I., Wu, F., Ourmanov, I., et al. (2013). Conformational epitope consisting of the V3 and V4 loops as a target for potent and broad neutralization of simian immunodeficiency viruses. J. Virol. 87, 5424-5436. doi: 10.1128/JVI.00201-13

Kwon, Y. D., Finzi, A., Wu, X., Dogo-Isonagie, C., Lee, L. K., Moore, L. R., et al. (2012). Unliganded HIV-1 gp120 core structures assume the CD4bound conformation with regulation by quaternary interactions and variable loops. Proc. Natl. Acad. Sci. U.S.A. 109, 5663-5668. doi: 10.1073/pnas.11123 91109

Laird, M. E., Igarashi, T., Martin, M. A., and Desrosiers, R. C. (2008). Importance of the V1/V2 loop region of simian-human immunodeficiency virus envelope glycoprotein gp120 in determining the strain specificity of the neutralizing antibody response. J. Virol. 82, 11054-11065. doi: 10.1128/JVI.01341-08

Lebkowski, J. S., Clancy, S., and Calos, M. P. (1985). Simian virus 40 replication in adenovirus-transformed human cells antagonizes gene expression. Nature 317, 169-171. doi: 10.1038/317169a0

Lee, S., Peden, K., Dimitrov, D. S., Broder, C. C., Manischewitz, J., Denisova, G., et al. (1997). Enhancement of human immunodeficiency virus type 1 envelopemediated fusion by a CD4-gp120 complex-specific monoclonal antibody. J. Virol. 71, 6037-6043.

Lindorff-Larsen, K., Piana, S., Palmo, K., Maragakis, P., Klepeis, J. L., Dror, R. O., et al. (2010). Improved side-chain torsion potentials for the Amber ff99SB protein force field. Proteins 78, 1950-1958. doi: 10.1002/prot.22711

Liu, J., Bartesaghi, A., Borgnia, M. J., Sapiro, G., and Subramaniam, S. (2008). Molecular architecture of native HIV-1 gp120 trimers. Nature 455, 109-113. doi: $10.1038 /$ nature 07159

Lobritz, M. A., Marozsan, A. J., Troyer, R. M., and Arts, E. J. (2007). Natural variation in the $\mathrm{V} 3$ crown of human immunodeficiency virus type 1 affects replicative fitness and entry inhibitor sensitivity. J. Virol. 81, 8258-8269. doi: 10.1128/JVI.02739-06

Lusso, P., Earl, P. L., Sironi, F., Santoro, F., Ripamonti, C., Scarlatti, G., et al. (2005). Cryptic nature of a conserved, CD4-inducible V3 loop neutralization epitope in the native envelope glycoprotein oligomer of CCR5-restricted, but not CXCR4using, primary human immunodeficiency virus type 1 strains. J. Virol. 79, 6957-6968. doi: 10.1128/JVI.79.11.6957-6968.2005

Lyumkis, D., Julien, J. P., de Val, N., Cupo, A., Potter, C. S., Klasse, P. J., et al. (2013). Cryo-EM structure of a fully glycosylated soluble cleaved HIV-1 envelope trimer. Science 342, 1484-1490. doi: 10.1126/science.1245627

Mascola, J. R., and Haynes, B. F. (2013). HIV-1 neutralizing antibodies: understanding nature's pathways. Immunol. Rev. 254, 225-244. doi: 10.1111/imr.12075

McLellan, J. S., Pancera, M., Carrico, C., Gorman, J., Julien, J. P., Khayat, R., et al. (2011). Structure of HIV-1 gp120 V1/V2 domain with broadly neutralizing antibody PG9. Nature 480, 336-343. doi: 10.1038/nature10696

Moore, J. P., Sattentau, Q. J., Klasse, P. J., and Burkly, L. C. (1992). A monoclonal antibody to CD4 domain 2 blocks soluble CD4-induced conformational changes in the envelope glycoproteins of human immunodeficiency virus type 1 (HIV-1) and HIV-1 infection of CD4+ cells. J. Virol. 66, 4784-4793. 
Munro, J. B., Gorman, J., Ma, X., Zhou, Z., Arthos, J., Burton, D. R., et al. (2014). Conformational dynamics of single HIV-1 envelope trimers on the surface of native virions. Science 346, 759-763. doi: 10.1126/science.1254426

Naganawa, S., Yokoyama, M., Shiino, T., Suzuki, T., Ishigatsubo, Y., Ueda, A., et al. (2008). Net positive charge of HIV-1 CRF01_AE V3 sequence regulates viral sensitivity to humoral immunity. PLoS ONE 3:e3206. doi: 10.1371/journal.pone.0003206

Nomaguchi, M., Doi, N., Fujiwara, S., Saito, A., Akari, H., Nakayama, E. E., et al. (2013a). Systemic biological analysis of the mutations in two distinct HIV$1 \mathrm{mt}$ genomes occurred during replication in macaque cells. Microbes Infect. 15, 319-328. doi: 10.1016/j.micinf.2013.01.005

Nomaguchi, M., Yokoyama, M., Kono, K., Nakayama, E. E., Shioda, T., Doi, N., et al. (2013b). Generation of rhesus macaque-tropic HIV-1 clones that are resistant to major anti-HIV-1 restriction factors. J. Virol. 87, 11447-11461. doi: 10.1128/JVI.01549-13

Nomaguchi, M., Doi, N., Kamada, K., and Adachi, A. (2008). Species barrier of HIV-1 and its jumping by virus engineering. Rev. Med. Virol. 18, 261-275. doi: $10.1002 /$ rmv.576

Nomaguchi, M., Miyake, A., Doi, N., Fujiwara, S., Miyazaki, Y., TsunetsuguYokota, Y., et al. (2014). Natural single-nucleotide polymorphisms in the 3' region of the HIV-1 pol gene modulate viral replication ability. J. Virol. 88, 4145-4160. doi: 10.1128/JVI.01859-13

Ogert, R. A., Lee, M. K., Ross, W., Buckler-White, A., Martin, M. A., and Cho, M. W. (2001). N-linked glycosylation sites adjacent to and within the V1/V2 and the V3 loops of dualtropic human immunodeficiency virus type 1 isolate DH12 gp120 affect coreceptor usage and cellular tropism. J. Virol. 75, 59986006. doi: 10.1128/JVI.75.13.5998-6006.2001

Pacheco, B., Basmaciogullari, S., Labonte, J. A., Xiang, S. H., and Sodroski, J. (2008). Adaptation of the human immunodeficiency virus type 1 envelope glycoproteins to new world monkey receptors. J. Virol. 82, 346-357. doi: 10.1128/JVI.01299-07

Palker, T. J., Clark, M. E., Langlois, A. J., Matthews, T. J., Weinhold, K. J., Randall, R. R., et al. (1988). Type-specific neutralization of the human immunodeficiency virus with antibodies to env-encoded synthetic peptides. Proc. Natl. Acad. Sci. U.S.A. 85, 1932-1936. doi: 10.1073/pnas.85.6.1932

Pancera, M., Majeed, S., Ban, Y. E., Chen, L., Huang, C. C., Kong, L., et al. (2010). Structure of HIV-1 gp120 with gp41-interactive region reveals layered envelope architecture and basis of conformational mobility. Proc. Natl. Acad. Sci. U.S.A. 107, 1166-1171. doi: 10.1073/pnas.0911004107

Pancera, M., Zhou, T., Druz, A., Georgiev, I. S., Soto, C., Gorman, J., et al. (2014). Structure and immune recognition of trimeric pre-fusion HIV-1 Env. Nature 514, 455-461. doi: 10.1038/nature13808

Pinter, A., Honnen, W. J., He, Y., Gorny, M. K., Zolla-Pazner, S., and Kayman, S. C. (2004). The V1/V2 domain of gp120 is a global regulator of the sensitivity of primary human immunodeficiency virus type 1 isolates to neutralization by antibodies commonly induced upon infection. J. Virol. 78, 5205-5215. doi: 10.1128/JVI.78.10.5205-5215.2004

Recordon-Pinson, P., Raymond, S., Bellecave, P., Marcelin, A. G., Soulie, C., Descamps, D., et al. (2013). HIV-1 dynamics and coreceptor usage in Maraviroc-treated patients with ongoing replication. Antimicrob. Agents Chemother. 57, 930-935. doi: 10.1128/AAC.02159-12

Reeves, J. D., Gallo, S. A., Ahmad, N., Miamidian, J. L., Harvey, P. E., Sharron, M., et al. (2002). Sensitivity of HIV-1 to entry inhibitors correlates with envelope/coreceptor affinity, receptor density, and fusion kinetics. Proc. Natl. Acad. Sci. U.S.A. 99, 16249-16254. doi: 10.1073/pnas.252469399

Rusche, J. R., Javaherian, K., McDanal, C., Petro, J., Lynn, D. L., Grimaila, R., et al. (1988). Antibodies that inhibit fusion of human immunodeficiency virusinfected cells bind a 24-amino acid sequence of the viral envelope, gp120. Proc. Natl. Acad. Sci. U.S.A. 85, 3198-3202. doi: 10.1073/pnas.85.9.3198

Ryckaert, J.-P., Ciccotti, G., and Berendsen, H. J. C. (1977). Numerical integration of the cartesian equations of motion of a system with constraints: molecular dynamics of n-alkanes. J. Comput. Phys. 23, 327-341. doi: 10.1016/00219991(77)90098-5
Sato, H., Shiino, T., Kodaka, N., Taniguchi, K., Tomita, Y., Kato, K., et al. (1999). Evolution and biological characterization of human immunodeficiency virus type 1 subtype E gp120 V3 sequences following horizontal and vertical virus transmission in a single family. J. Virol. 73, 3551-3559.

Shiino, T., Kato, K., Kodaka, N., Miyakuni, T., Takebe, Y., and Sato, H. (2000). A group of V3 sequences from human immunodeficiency virus type 1 subtype E non-syncytium-inducing, CCR5-using variants are resistant to positive selection pressure. J. Virol. 74, 1069-1078. doi: 10.1128/JVI.74.3.10691078.2000

Stamatatos, L., and Cheng-Mayer, C. (1995). Structural modulations of the envelope gp120 glycoprotein of human immunodeficiency virus type 1 upon oligomerization and differential V3 loop epitope exposure of isolates displaying distinct tropism upon virion-soluble receptor binding. J. Virol. 69, 6191-6198.

Sullivan, N., Thali, M., Furman, C., Ho, D. D., and Sodroski, J. (1993). Effect of amino acid changes in the V1/V2 region of the human immunodeficiency virus type 1 gp120 glycoprotein on subunit association, syncytium formation, and recognition by a neutralizing antibody. J. Virol. 67, 3674-3679.

Tasca, S., Ho, S. H., and Cheng-Mayer, C. (2008). R5X4 viruses are evolutionary, functional, and antigenic intermediates in the pathway of a simian-human immunodeficiency virus coreceptor switch. J. Virol. 82, 7089-7099. doi: 10.1128/JVI.00570-08

Tilton, J. C., and Doms, R. W. (2010). Entry inhibitors in the treatment of HIV-1 infection. Antiviral Res. 85, 91-100. doi: 10.1016/j.antiviral.2009.07.022

Troyer, R. M., Collins, K. R., Abraha, A., Fraundorf, E., Moore, D. M., Krizan, R. W., et al. (2005). Changes in human immunodeficiency virus type 1 fitness and genetic diversity during disease progression. J. Virol. 79, 9006-9018. doi: 10.1128/JVI.79.14.9006-9018.2005

von Schwedler, U., Song, J., Aiken, C., and Trono, D. (1993). Vif is crucial for human immunodeficiency virus type 1 proviral DNA synthesis in infected cells. J. Virol. 67, 4945-4955.

Wilen, C. B., Tilton, J. C., and Doms, R. W. (2012). HIV: cell binding and entry. Cold Spring Harb. Perspect. Med. 2, a006866. doi: 10.1101/cshperspect.a 006866

Willey, R. L., Smith, D. H., Lasky, L. A., Theodore, T. S., Earl, P. L., Moss, B., et al. (1988). In vitro mutagenesis identifies a region within the envelope gene of the human immunodeficiency virus that is critical for infectivity. J. Virol. 62, 139-147.

Yamashita, T., Doi, N., Adachi, A., and Nomaguchi, M. (2008). Growth ability in simian cells of monkey cell-tropic HIV-1 is greatly affected by downstream region of the vif gene. J. Med. Invest. 55, 236-240. doi: 10.2152/jmi.55.236

Yokoyama, M., Naganawa, S., Yoshimura, K., Matsushita, S., and Sato, H. (2012). Structural dynamics of HIV-1 envelope Gp120 outer domain with V3 loop. PLoS ONE 7:e37530. doi: 10.1371/journal.pone.0037530

Yuan, Y., Yokoyama, M., Maeda, Y., Terasawa, H., Harada, S., Sato, H., et al. (2013). Structure and dynamics of the gp120 V3 loop that confers noncompetitive resistance in R5 HIV-1(JR-FL) to maraviroc. PLoS ONE 8:e65115. doi: 10.1371/journal.pone.0065115

Zolla-Pazner, S., and Cardozo, T. (2010). Structure-function relationships of HIV-1 envelope sequence-variable regions refocus vaccine design. Nat. Rev. Immunol. 10, 527-535. doi: 10.1038/nri2801

Conflict of Interest Statement: The authors declare that the research was conducted in the absence of any commercial or financial relationships that could be construed as a potential conflict of interest.

Copyright (C) 2016 Yokoyama, Nomaguchi, Doi, Kanda, Adachi and Sato. This is an open-access article distributed under the terms of the Creative Commons Attribution License (CC BY). The use, distribution or reproduction in other forums is permitted, provided the original author(s) or licensor are credited and that the original publication in this journal is cited, in accordance with accepted academic practice. No use, distribution or reproduction is permitted which does not comply with these terms. 